\title{
Stability Analysis and Control of Rigid-Body Systems with Impacts and Friction
}

\author{
Michael Posa, Mark Tobenkin, and Russ Tedrake, Member, IEEE
}

\begin{abstract}
Many critical tasks in robotics, such as locomotion or manipulation, involve collisions between a rigid body and the environment or between multiple bodies. Methods based on sums-of-squares (SOS) for numerical computation of Lyapunov certificates are a powerful tool for analyzing the stability of continuous nonlinear systems, and can additionally be used to automatically synthesize stabilizing feedback controllers. Here, we present a method for applying sums-of-squares verification to rigid bodies with Coulomb friction undergoing discontinuous, inelastic impact events. The proposed algorithm explicitly generates Lyapunov certificates for stability, positive invariance, and safety over admissible (non-penetrating) states and contact forces. We leverage the complementarity formulation of contact, which naturally generates the semialgebraic constraints that define this admissible region. The approach is demonstrated on multiple robotics examples, including simple models of a walking robot, a perching aircraft, and control design of a balancing robot.
\end{abstract}

Index Terms-Lyapunov analysis and stability verification, control design, rigid-body dynamics with impacts and friction, sums-of-squares

\section{INTRODUCTION}

$\mathbf{M}$ ANY tasks in robotics require making and breaking contact with objects in the robot's environment. For highly dynamic tasks, such as walking [11], [60], and perching [12], this drives the need for control design techniques capable of handling impulsive dynamics and realistic friction models. Recent work has demonstrated that, for smooth nonlinear systems, techniques for stability verification can play a pivotal role in incremental motion planning and control design strategies, [53], and in direct optimization over feedback laws [18], [28]. Motivated by these developments, this paper presents a numerical approach for analyzing questions of stability, invariance, and safety for rigid-body systems subject to inelastic collisions and friction.

Our central observation is that the complementarity framework for modeling such systems (e.g. [6], [49], [22]) is compatible with recent advances in polynomial optimization, in particular sums-of-squares (SOS) optimization [37]. For a polynomial to be non-negative, it is sufficient that it be expressible as the sum of squares of polynomials. Optimizing over such polynomials can be formulated as a semidefinite program (SDP), which is a form of convex optimization. In the controls community, SOS has been widely applied, particularly in automating Lyapunov analysis of polynomial dynamical systems [15] and hybrid systems [42]. Here, we model the non-smooth and impulsive dynamics of systems

M. Posa, M. Tobenkin, and R. Tedrake are with the Department of Electrical Engineering and Computer Science, Massachusetts Institute of Technology, Cambridge, MA, 02139 e-mail: mposa@mit.edu, smarkmt@gmail.com, russt@mit.edu with frictional impacts via the complementarity framework. A major advantage of this framework over traditional approaches using hybrid automata is that the dynamics can be expressed without suffering from the combinatorial explosion of "modes" resulting from distinct combinations of contacts. Hybrid methods which must explicitly enumerate each mode and transition are fundamentally fated to scale poorly in the number of potential contacts. This approach also naturally encompasses instances of Zeno phenomena, which pose a challenge in some frameworks [2], [20]. Instead, we describe the dynamics with conditions expressed jointly in the coordinates, velocities and feasible contact forces. The size of the resulting SDP grows quadratically in the number of contact points.

This work demonstrates how Lyapunov analysis can be performed by testing sufficient semialgebraic conditions in these variables. We present algorithms based on testing these semialgebraic conditions using SOS optimization. Procedures are provided which solve convex programs (or a sequence of such programs) to maximize the size of regions of positive invariance or guaranteed regions of "safety". We are additionally interested in the design of mode-invariant feedback controllers-that is, control laws which are a smooth functions of state, rather than a switching or hybrid policies. Verified, mode-invariant control would be useful in providing robustness to disturbances which affect the contact state, or on systems with limited contact sensing. We apply the algorithms detailed here on a set of systems of interest to the robotics community.

\section{A. Related Work}

In this paper, we adopt the complementarity formulation for modeling rigid bodies with frictional impacts [56], [7]. This framework and its historical development are reviewed in [49] and a more comprehensive bibliography and discussion are provided in [6]. Notions of equilibria, stability, and extensions of Lyapunov analysis to such systems are presented in [6] and [22] (see also the related article [23]). The numerical structure of the complementarity framework has previously been exploited to avoid mode enumeration in the task of trajectory optimization [39]. Section II will summarize the aspects of these works used in this paper.

An alternative formalism for modeling and control of non-smooth mechanical systems is that of hybrid automata [5]. Example applications of this framework to the control and analysis of hybrid mechanical systems can be found in [27], [8], [47], and [35]. A number of numerical techniques have been presented for addressing verification, stability, and control design of general hybrid automata. These include 
methods based on approximate solutions of Hamilton-Jacobi equations, [54], and the construction of discrete abstractions [1]. Optimization has also been used to find polynomial barrier certificates, [41], [42], as well as Lyapunov functions about equilibria, [36], and of transverse dynamics about hybrid limit cycles [31].

These last three approaches share a common computational framework in that they employ sums-of-squares. In [36], numerical techniques were presented for finding certificates of stability for hybrid systems governed by polynomial vector fields (both external switching and polynomial guards were examined). The work in [42] examined safety verification for both deterministic and stochastic systems. The fundamental difference between the approaches in [42], [36], and [31] and in this work stems from the choice of modeling framework. These previous works require explicit enumeration of a set of "modes," in which the system is subject to a particular differential equation or inclusion and conditions under which these modes switch. When modeling a Lagrangian mechanical system with $m$ potential contact points, there may be as many as $3^{m}$ distinct modes related to the contact-free, sliding, and sticking cases. By contrast, this work uses a complementarity model and simultaneously reasons over the set of system trajectories and feasible contact force trajectories. In doing so, we exploit the inherent structure of rigid-body dynamics and avoid this combinatorial mode enumeration.

A preliminary version of this paper was presented at the 2013 Hybrid Systems: Computation and Control (HSCC) conference [40]. Here, we improve on the previous algorithms by separating the effects of the contact forces (see Theorem 4) and extending the previous work to include control design via sums-of-squares. The updated algorithm also noticeably increased the size of the verified region of the simple walking model. We provide a more complete and rigorous analysis of the method and background material and demonstrate the proposed algorithm on a seven state system along with the automatic synthesis of a feedback controller.

\section{BACKGROUND}

Here, we introduce a notion of solutions to discontinuous rigid-body systems and describe the friction and impact laws used in this paper.

\section{A. Measure Differential Inclusions}

We consider systems whose state is given by a set of generalized coordinates $q \in \mathbb{R}^{n}$ and generalized velocities $v \in \mathbb{R}^{n}$ and we let $x=\left[q^{T} v^{T}\right]^{T}$. For mechanical systems, $q(t)$ will evolve continuously whereas $v(t)$ may have discontinuities due to impacts, which present an obstacle to applying classical Lyapunov analysis.

Recently, a number of authors have provided extensions of Lyapunov analysis to discontinuous dynamics using the framework of measure differential inclusions (MDIs), first introduced by Moreau [33], with additional details and precise definitions in [22] and [6]. By taking the time-derivative of state from a set-valued (and not necessarily bounded) function, MDIs address both the discontinuities and non-smoothness of the system evolution that arise from impacts and standard friction force laws. We provide a high-level overview of MDIs here, focused on autonomous Lagrangian mechanical systems.

A solution of a measure differential inclusion will be taken to be a pair of functions, $q(t)$ and $v(t)$, such that $q(t)$ is absolutely continuous and $v(t)$ is of locally bounded variation (LBV), allowing for countably many discontinuities. The left and right limits of $v(t)$, denoted $v^{-}(t)$ and $v^{+}(t)$, are guaranteed to exist and we require that solutions satisfy:

$$
\begin{aligned}
q(t)-q\left(t_{0}\right) & =\int_{t_{0}}^{t} v(\tau) d \tau \\
v^{+}(t)-v^{-}\left(t_{0}\right) & =\int_{t_{0}}^{t} \dot{v}(\tau) d \tau+\int_{t_{0}}^{t} v^{+}(\tau)-v^{-}(\tau) d \eta(\tau) .
\end{aligned}
$$

Here $\dot{v}(t)$ is an integrable function and $\eta$ is a sum of Dirac measures centered at times $\left\{t_{k}\right\}_{k=1}^{\infty}$, which model the continuous evolution and jumps in the velocity respectively. By assumption, $v(t)$ has no singular part (see [22], Ch. 3). To describe the dynamics, we must give rules for specifying legal values of $\dot{v}(t)$, the locations $\left\{t_{k}\right\}_{k=1}^{\infty}$, and the jumps $v^{+}\left(t_{k}\right)-$ $v^{-}\left(t_{k}\right)$. Specific rules are given in the next section, but we briefly note the following: we require $\dot{v}(t) \in \mathfrak{F}(q(t), v(t))$ for almost all $t$, where $\mathfrak{F}(q, v)$ is a set of possible values. This use of differential inclusions rather than equations is a standard approach for addressing Coulomb friction and similar phenomena. Similarly, the value of jumps will be drawn from a set which generally depends on $q(t)$ and $v^{-}(t)$. The locations of impacts will be defined implicitly by the locations where $v^{+}(t)$ and $v^{-}(t)$ disagree. Finally, we take $v(t)$ to be undefined at points of discontinuity.

Our problems center around systems where solutions must lie in an admissible set, $\mathcal{A}$, defined by a finite family of functions $\phi_{i}: \mathbb{R}^{n} \rightarrow \mathbb{R}:$

$$
\mathcal{A}=\left\{(q, v) \in \mathbb{R}^{2 n} \mid \phi_{i}(q) \geq 0 \forall i \in\{1, \ldots, m\}\right\} .
$$

Here, the functions $\phi_{i}(\cdot)$ are gap functions, representing nonpenetration constraints for rigid bodies. $\phi_{i}(q)<0$ indicates that configuration $q$ is in penetration and $\phi_{i}(q)=0$ is equivalent to contact at point $i$. We will focus on MDIs which are consistent (see [22], Ch. 4).

Definition 1. A measure differential inclusion is consistent if every solution defined for $t_{0}$ is defined for almost all $t \geq t_{0}$, all such solutions remain within $\mathcal{A}$, and for each $x_{0} \in \mathcal{A}$ there exists at least one solution passing through $x_{0}$.

An equilibrium point for such a system is defined as any point $x_{0} \in \mathcal{A}$ such that $x(t)=x_{0}$ is a solution. As is typical for models with dry friction, we do not expect to have unique solutions from the systems covered by this work [6], [49]. For systems governed by MDIs, there are natural extensions to the notions of stability and positive invariance ([22] Ch. 6).

Definition 2. An equilibrium point $x_{0} \in \mathcal{A}$ of a consistent MDI is stable in the sense of Lyapunov if, for each $\epsilon>0$, there exists a $\delta>0$ such that every solution $x(t)$ satisfying $\left|x_{0}-x\left(t_{0}\right)\right|<\delta$ satisfies $\left|x_{0}-x(t)\right|<\epsilon$ for almost all $t \geq t_{0}$. 
Definition 3. $A$ set $B \subset \mathcal{A}$ is positively invariant if each solution $x(t)$ satisfying $x^{-}\left(t_{0}\right) \in B$ satisfies $x(t) \in B$ for almost all $t \geq t_{0}$.

In order to apply Lyapunov analysis to MDIs, we make note of the following fact (see [22], Proposition 6.3): if a function $V: D \rightarrow \mathbb{R}$ is a continuously differentiable function on a compact set $D \subset \mathbb{R}^{2 n}$, and $x(t)$ is LBV, then $V(x(t))$ is also LBV and

$$
\begin{aligned}
V\left(x^{+}(t)\right)-V\left(x^{-}\left(t_{0}\right)\right) & =\int_{t_{0}}^{t} \frac{\partial V}{\partial x} \dot{x}(\tau) d \tau \\
& +\int_{t_{0}}^{t} V\left(x^{+}(\tau)\right)-V\left(x^{-}(\tau)\right) d \eta(\tau),
\end{aligned}
$$

where $\dot{x}(t)=\left[v(t)^{T} \dot{v}(t)^{T}\right]^{T}$, as in (1) and (2). For the remainder of this paper, when we write $\mathrm{d} V(x) \leq 0$ for certain $x \in A$ we mean that, for any solution satisfying $x^{-}(t)=x$, we have $\frac{\partial V}{\partial x} \dot{x}(t) \leq 0$ and $V\left(x^{+}(t)\right)-V\left(x^{-}(t)\right) \leq 0$.

\section{B. Rigid-Body Dynamics}

Many robotic systems are appropriately modeled as a set of rigid links connected through some combination of joints. The continuous dynamics of these rigid-body systems subject to frictional forces can be modeled by the manipulator equations

$$
H(q) \dot{v}+C(q, v)=J(q)^{T} \lambda_{N}+J_{f}(q)^{T} \lambda_{T},
$$

where the dependence of $q, v, \lambda_{N}$, and $\lambda_{T}$ on time has been suppressed for clarity. Here, $H(q)$ is the inertia matrix and $C(q, v)$ is the combined Coriolis and gravitational terms. For $m$ potential contacts, the constraint forces are split into those normal to the contacts, $\lambda_{N} \in \mathbb{R}^{m}, \lambda_{N} \geq 0$, and the frictional forces tangential to the contact surface, $\lambda_{T}$. For planar systems, $\lambda_{T} \in \mathbb{R}^{m}$ while $\lambda_{T} \in \mathbb{R}^{2 m}$ in the full three dimensional case. We will also write $\lambda$ to be the stacked vector $\left[\begin{array}{cc}\lambda_{N}^{T} & \lambda_{T}^{T}\end{array}\right]^{T}$. The matrices $J(q) \in \mathbb{R}^{m \times n}$ and $J_{f}(q) \in \mathbb{R}^{m \times n}$ (in the planar case) project the normal and frictional contact forces into joint coordinates. We will also refer to $J_{i}(q)$ and $J_{f, i}(q)$ as the $i$ th row of $J$ and $J_{f}$, associated with the particular contact forces $\lambda_{N, i}$ and $\lambda_{T, i}$.

We will focus on the dynamics of rigid bodies interacting at a fixed number of contact points, though these points may not necessarily be fixed on their respective bodies. When $\phi(q)$ represents the distances between pairs of bodies, we can take $J$ to be the Jacobian $\frac{\partial \phi}{\partial q}$ and $J_{f}$ defines the frictional plane. Here, the velocity of the $i$ th contact point has components $J_{i} v(t)$ and $J_{f, i} v(t)$ normal to and tangential to the contact surface. We use a simple Coulomb friction model to represent our contact forces:

$$
\begin{aligned}
J_{f, i} v(t)=0 & \Rightarrow\left\|\lambda_{T, i}(t)\right\| \leq \mu \lambda_{N, i}(t), \\
J_{f, i} v(t) \neq 0 & \Rightarrow \lambda_{T, i}(t)=-\frac{J_{f, i} v(t)}{\left\|J_{f, i} v(t)\right\|} \mu \lambda_{N, i}(t) .
\end{aligned}
$$

When the tangential velocity vanishes, $\lambda_{T, i}$ can take on any value within the friction cone. If the contact point is sliding, then $\lambda_{T, i}$ directly opposes the direction of slip.

\section{Inelastic Collisions with Friction}

Rigid-body impacts are often modeled as instantaneous events where an impulse causes a discontinuity in the velocity. Impacts occur when there is contact, $\phi_{i}(q(t))=0$, and when consistency requires an instantaneous change in velocity to prevent penetration. As with the continuous case, we let $\Lambda_{N}, \Lambda_{T}$ be the normal and tangential impulses. Derived from the manipulator equations, the pre- and post-impact velocities for a collision at the $i$ th contact point are related by $v^{+}(t)=v^{-}(t)+H^{-1}\left(J_{i}^{T} \Lambda_{N, i}+J_{f, i}^{T} \Lambda_{T, i}\right)$. In the special case of a single, frictionless inelastic collision, we observe that the inelastic condition is $J_{i} v^{+}(t)=0$ and so we can explicitly solve for the normal impulse and post-impact state:

$$
\begin{aligned}
\Lambda_{N, i} & =-\left(J_{i} H^{-1} J_{i}^{T}\right)^{-1} J_{i} v^{-}(t), \\
v^{+}(t) & =\left(I-H^{-1} J_{i}^{T}\left(J_{i} H^{-1} J_{i}^{T}\right)^{-1} J_{i}\right) v^{-}(t) .
\end{aligned}
$$

However, when considering Coulomb friction, we have no explicit formula. To model frictional collisions, we adopt an impact law first proposed by Routh [44] and described in detail in [59], [4]. Originally a graphical approach, this method constructs a path in impulse space that will fit naturally into our Lyapunov analysis. To briefly summarize Routh's technique for computing the net impulses and the post-impact state:

1) Monotonically increase the normal impulse $\Lambda_{N, i}$ with slope $\Lambda_{N, i}^{\prime}$.

2) Increment the tangential impulse $\Lambda_{T, i}$ with slope $\Lambda_{T, i}^{\prime}$, according to the friction law:

$$
\begin{aligned}
J_{f, i} \bar{v}=0 & \Rightarrow\left\|\Lambda_{T, i}^{\prime}\right\| \leq \mu \Lambda_{N, i}^{\prime}, \\
J_{f, i} \bar{v} \neq 0 & \Rightarrow \Lambda_{T, i}^{\prime}=-\frac{J_{f, i} \bar{v}}{\left\|J_{f, i} \bar{v}\right\|} \mu \Lambda_{N, i}^{\prime}
\end{aligned}
$$

where $\bar{v}=v^{-}(t)+H^{-1}\left(J_{i}^{T} \Lambda_{N, i}+J_{f, i}^{T} \Lambda_{T, i}\right)$ is the velocity after impulses $\Lambda_{N, i}$ and $\Lambda_{T, i}$.

$3)$ Terminate when the normal contact velocity vanishes, $J_{i} \bar{v}=0$, and take $v^{+}(t)=\bar{v}$.

This method amounts to following continuous path in the impulse space where the slopes of the impulses are $\Lambda_{N, i}^{\prime}$ and $\Lambda_{T, i}^{\prime}$. Diagrams showing the resolution of two potential planar impacts are shown in Figure 1. Along each linear section, these slopes must satisfy the Coulomb friction constraints. Solutions may transition from sliding to sticking and vice versa and the direction of slip may even reverse as a result of each impact. Note, while this path is piecewise linear in the planar case, this is not true in three dimensions.

1) Simultaneous Impacts: The question of simultaneous impacts has been well studied in both simulation and analysis [9], but remains a difficult problem. Here, since we are ultimately interested in the question of stability, we take a permissive view of simultaneous impacts. If the set $\mathcal{J} \subset\{1, \ldots, m\}$ is the set of active impacts $\left(\phi_{i}=0, J_{i} \bar{v} \leq 0\right)$, then we proceed with Routh's method where the incremental impulses are any arbitrary convex combination of the incremental impulses for 


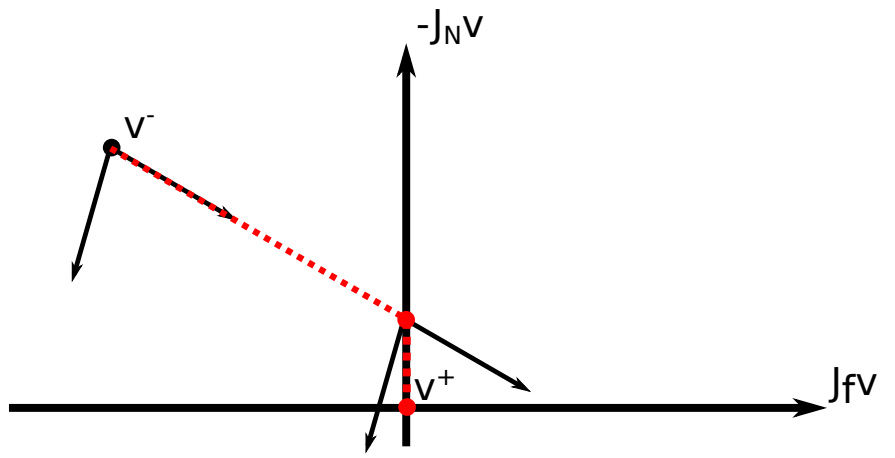

(a) The contact velocity, in the contact frame, is plotted throughout an impact resolution by Routh's method. At the initial state, $v^{-}$, the extreme rays of the friction cone are shown as solid arrows. Since the contact is sliding, $J_{f} v<0$, the impulse increments along the appropriate ray. When $v(s)$, shown in the dotted line, intersects the $J_{f} v=0$ axis, the contact transitions to sticking and the impact terminates when $J_{N} v(s)=0$.

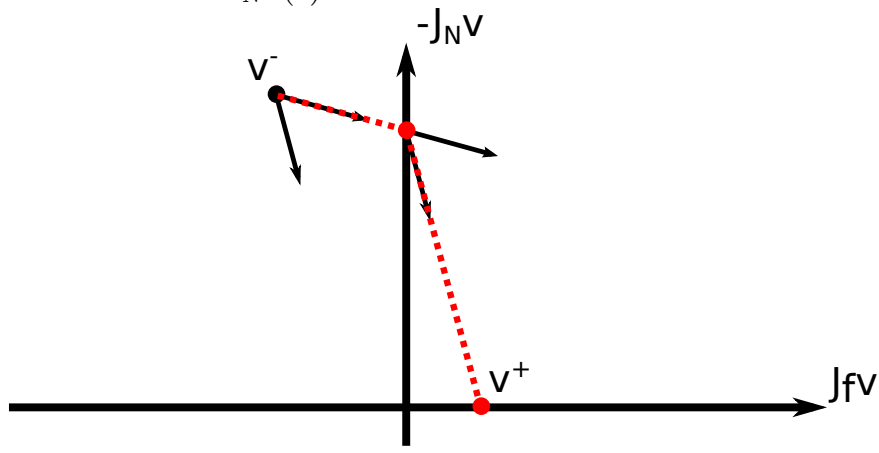

(b) In this case, the impulse required to maintain sticking is not within the friction cone. Therefore, when $v(s)$ crosses the vertical axis, the contact does not stick, but the direction of slip reverses, and the impulse increments along the other extreme ray until termination.

Fig. 1: Example impact resolutions via Routh's method.

each impact. That is, where $e_{i}$ are the standard basis vectors,

$$
\begin{aligned}
\Lambda_{N}^{\prime} & =\sum_{i \in \mathcal{J}} e_{i} \Lambda_{N, i}^{\prime} \\
\Lambda_{f}^{\prime} & =\sum_{i \in \mathcal{J}} e_{i} \Lambda_{f, i}^{\prime} \\
1 & =\sum_{i \in \mathcal{J}} \Lambda_{N, i}^{\prime} .
\end{aligned}
$$

With this model, we allow the collisions to be resolved simultaneously or in an arbitrary order. Additionally, we will later show that this choice of model does not introduce any complexity to the problem of Lyapunov analysis.

\section{CONDitions FOR STABILITY}

The highly-structured nature of rigid-body dynamics and the complementarity formulation of contact allow us to construct semialgebraic conditions for stability in the sense of Lyapunov and positive invariance.

\section{A. Lyapunov Conditions for MDIs}

We begin by describing sufficient conditions for stability in the sense of Lyapunov and positive invariance, stated in terms of Lyapunov functions. Recall that a function $\alpha:[0, \infty) \rightarrow$ $[0, \infty)$ belongs to class $\mathcal{K}$ if it is strictly increasing and $\alpha(0)=$ 0 . The following theorem is adapted from [22], Theorem 6.23, and is stated without proof.

Theorem 1. Let $x_{0}$ be an equilibrium point for a consistent $M D I$ and let $V: \mathbb{R}^{2 n} \rightarrow \mathbb{R}$ be a continuously differentiable function. If there exists a neighborhood $U$ of $x_{0}$ and a class $\mathcal{K}$ function $\alpha$ such that $x \in U \cap \mathcal{A}$ implies $\mathrm{d} V \leq 0$ and $V(x) \geq \alpha\left(\left\|x-x_{0}\right\|\right)$ then $x_{0}$ is stable in the sense of Lyapunov.

For a candidate Lyapunov function $V(q, v)$, define the $c$ sublevel set

$$
\Omega_{c}=\left\{(q, v) \in \mathbb{R}^{2 n} \mid V(q, v)<c\right\}
$$

For a system whose solutions are continuous functions of time, $\mathrm{d} V \leq 0$ on $\Omega_{c} \cap \mathcal{A}$ would be sufficient to show that each connected component of $\Omega_{c} \cap \mathcal{A}$ is positively invariant. However, where $v(t)$ is discontinuous, the pre- and post-impact states may be in disjoint connected components. The following theorem provides stronger conditions which guarantee positive invariance of such a connected component. The proof can be found in Appendix A.

Theorem 2. Let $V: \mathbb{R}^{2 n} \rightarrow \mathbb{R}$ be a continuously differentiable function, and $\mathcal{C}$ be a connected component of $\Omega_{c} \cap \mathcal{A}$ with $\mathrm{d} V \leq 0$ on $\mathcal{C}$. Then $\mathcal{C}$ is positively invariant if, for every solution $(q(t), v(t))$ with $\left(q(t), v^{-}(t)\right) \in \mathcal{C}$, there exists a path $\bar{v}(s)$ from $v^{-}(t)$ to $v^{+}(t)$ such that $V(q(t), \bar{v}(s))$ is a nonincreasing function of $s$.

Theorem 2 holds for general Lyapunov functions and systems where the unilateral constraints are defined by the generalized positions $q$. While we are most often interested in systems with friction, we briefly consider the special structure implied by rigid-body dynamics and frictionless, inelastic collisions. The following proposition, whose proof is in Appendix B, shows that for such systems, and if $V$ is a convex function in $v$ for each fixed $q$, the above sufficient condition for positive invariance is also necessary. In particular, no additional conservatism is added by requiring $\bar{v}$ to be the chord connecting $v^{-}(t)$ to $v^{+}(t)$.

Proposition 3. For a rigid-body system undergoing frictionless, inelastic impacts, let $V: \mathbb{R}^{2 n} \rightarrow \mathbb{R}$ be a continuously differentiable function, and $\mathcal{C}$ be a connected component of $\Omega_{c} \cap \mathcal{A}$ such that $\mathrm{d} V \leq 0$ on $\mathcal{C}$. If $V$ is convex in $v$ for each fixed $q$ the following conditions are equivalent:

(i) $\mathcal{C}$ is positively invariant.

(ii) For each solution $(q(t), v(t))$, when $\left(q(t), v^{-}(t)\right) \in \mathcal{C}$, $V(q(t), \bar{v}(s))$ is non-increasing along the path $\bar{v}(s)=$ $s v^{+}(t)+(1-s) v^{-}(t)$ for $s \in[0,1]$

The proof for Proposition 3 fails for contacts with friction since the Routh path from pre- to post-impact states is no longer a line segment. The frictionless assumption does, however, cover a class of interesting problems including collisions due to impacting hard joint limits. 


\section{B. Conditions For Complementarity Systems}

We now focus on Lagrangian mechanical systems with impacts and friction described by complementarity conditions. This section contains sufficient conditions for demonstrating $\mathrm{d} V \leq 0$ and the additional constraint on jump discontinuities in the statement of Theorem 2. We partition the admissible set $\mathcal{A}$ into three disjoint sets: $\mathcal{F}, \mathcal{I}$, and $\mathcal{U}$.

$\mathcal{F}=\left\{(q, v) \in \mathcal{A}: \phi_{i}(q)=0 \Rightarrow J_{i}(q) v>0 \forall i \in\{1, \ldots, m\}\right\}$

$\mathcal{I}=\left\{(q, v) \in \mathcal{A}: \exists i \in\{1, \ldots, m\} \quad \phi_{i}(q)=0, J_{i}(q) v<0\right\}$

$\mathcal{U}=\left\{(q, v) \in \mathcal{A} \backslash \mathcal{I}: \exists i \in\{1, \ldots, m\} \quad \phi_{i}(q)=0, J_{i}(q) v=0\right\}$

Intuitively, $\mathcal{F}$ is the region where there is no contact or all contacts are being broken, so all the contact forces must vanish. On $\mathcal{F}$, we know that $\lambda=\Lambda=0$ and so that $\mathrm{d} V \leq 0$ is equivalent to

$$
\nabla V(q, v)^{T}\left[\begin{array}{c}
v \\
-H^{-1} C
\end{array}\right] \leq 0 .
$$

On $\mathcal{U}$, there may be forces due to contact and the condition on continuous state evolution is

$$
\nabla V(q, v)^{T}\left[\begin{array}{c}
v \\
-H^{-1}\left(C+J^{T} \lambda_{N}+J_{f}^{T} \lambda_{f}\right)
\end{array}\right] \leq 0 .
$$

On $\mathcal{I}$, there must be a collision for a trajectory to remain within $\mathcal{A}$. Additionally, consistency may also require collisions on $\mathcal{U}$ (for a detailed explanation of this, see the discussion of Painlevé's paradox in [49]). We now provide conditions on $V$ and a path for each jump discontinuity such that the requirements of Theorem 2 are satisfied. Furthermore, this path ensures that the jump conditions for $\mathrm{d} V \leq 0$ are met. We implicitly define the path between pre- and post-impact states. In the frictionless case, this path corresponds directly to the path in Proposition 3. Recall that the Routh method of Section II-C constructs a path through the space of contact impulses. We take $\bar{v}(s)$ to be the velocities defined in step 2 of the Routh method, where $s$ is the path parameter varying the impulses. As $\bar{v}$ depends linearly on the forces, we can find the derivative of $\bar{v}$ with respect to $s$, defined almost everywhere along each path segment:

$$
\frac{d \bar{v}(s)}{d s}=H^{-1}\left(J_{i}^{T} \Lambda_{N, i}^{\prime}+J_{f, i}^{T} \Lambda_{T, i}^{\prime}\right),
$$

where $\Lambda_{N, i}^{\prime}$ and $\Lambda_{T, i}^{\prime}$ satisfy the Coulomb friction conditions. To show $V$ is non-increasing along the path, we require

$$
\left.\frac{\partial V(q, v)}{\partial v}\right|_{(q, \bar{v}(s))} H^{-1}\left(J_{i}^{T} \Lambda_{N, i}^{\prime}+J_{f, i}^{T} \Lambda_{T, i}^{\prime}\right) \leq 0 .
$$

Since the Routh method for resolving impacts is memoryless, any point $(q, \bar{v}(s))$ is also a possible pre-impact state. So the set of all possible $(q, \bar{v}(s))$ is precisely equivalent to $\mathcal{A} \backslash \mathcal{F}$ and it is equivalent to enforce (11) for all $(q, v) \in \mathcal{A} \backslash \mathcal{F}$ instead of along potential paths. This constraint must hold for all $i$, so we construct a single condition that encompasses all contact points:

$$
\frac{\partial V(q, v)}{\partial v} H^{-1}\left(J^{T} \Lambda_{N}^{\prime}+J_{f}^{T} \Lambda_{T}^{\prime}\right) \leq 0 .
$$

Both (10) and (12) are defined in terms of permissible contact forces $\lambda$ and slopes of the impulse path $\Lambda^{\prime}$ when resolving collisions. Complementarity conditions can be used to describe the set of feasible contact normal forces [6], [49], [22]:

$$
\begin{aligned}
\phi_{i}, \lambda_{N, i} & \geq 0, \\
\phi_{i} \lambda_{N, i} & =0, \\
\left(J_{i} v\right) \lambda_{N, i} & \leq 0 .
\end{aligned}
$$

These constraints prohibit contact at a distance and ensure that the contact normal is a compressive and dissipative force. Note that (13-15) apply not only to the continuous force $\lambda$, but they also describe the set of feasible impulse slopes $\Lambda^{\prime}$. Observing that the friction constraints on both are also identical, we write the additional set of constraints:

$$
\begin{aligned}
\left(J_{f, i} v\right) \lambda_{T, i} & \leq 0, \\
\mu^{2} \lambda_{N, i}^{2}-\lambda_{T, i}^{2} & \geq 0, \\
\left(\mu^{2} \lambda_{N, i}^{2}-\lambda_{T, i}^{2}\right)\left(J_{f, i} v\right) & =0 .
\end{aligned}
$$

Here, we describe the proper nonlinear friction cone and diverge from the standard linear complementarity description of Coulomb friction. However, for any $(q, v)$, this full set of conditions is exactly equivalent to our formulations of both frictional, inelastic collisions and Coulomb friction.

\section{Separability of Contacts}

We now have three separate positivity conditions for stability. We require (9) to hold on $\mathcal{F}$, (10) on $\mathcal{U}$, and (12) on $\mathcal{U} \cup \mathcal{I}$, with the contact forces and impulses subject to (13) - (18). This formulation has the issue that the $\mathcal{O}(m)$ contact force terms, when appearing together, will significantly increase the size of verification programs we formulate in Section IV, detailed in Section IV-E. However, the structure in the problem leads to a significant reduction in complexity. For this, we require an additional assumption, that the contact surfaces themselves are distinguishable from one another. This rather benign assumption is satisfied by most rigid-body systems of interest, even including situations where "jamming" contact may occur.

Assumption 1. For all $i \in\{1, \ldots, m\}$ and for any $(q, v)$ where $\phi_{i}(q)=0$ and $J_{i} v \leq 0$, there exists a sequence $\left(q_{k}, v_{k}\right) \rightarrow$ $(q, v)$ where

1) $\phi_{i}\left(q_{k}\right)=0$ and $J_{i} v_{k} \leq 0$ and

2) $\phi_{j}\left(q_{k}\right)>0$ or $J_{j} v_{k}>0$ for all $j \neq i$.

Theorem 4. Given Assumption $1, \mathrm{~d} V \leq 0$ is equivalent to requiring (9) to hold on $\mathcal{F}$, and

$$
\frac{\partial V(q, v)}{\partial v} H^{-1}\left(J_{i}^{T}+J_{f, i}^{T} \lambda_{T, i}\right) \leq 0, \forall i=1, \ldots, m
$$

whenever the following conditions hold:

$$
\begin{aligned}
\phi_{i} & =0, \\
J_{i} v & \leq 0, \\
\left(J_{f, i} v\right) \lambda_{T, i} & \leq 0, \\
\mu^{2}-\lambda_{T, i}^{2} & \geq 0, \\
\left(\mu^{2}-\lambda_{T, i}^{2}\right)\left(J_{f, i} v\right) & =0, \\
\phi_{j} & \geq 0, \forall j \neq i .
\end{aligned}
$$


Proof. First, observe that the original conditions on $\lambda$ and $\Lambda$ are identical, and they appear in distinct conditions and constraints, so we may combine them and treat the two simply as $\lambda$. To show that these conditions are sufficient is straightforward, and does not require Assumption 1. Since (13) - (18) and (12) are homogeneous in $\left(\lambda_{N, i}, \lambda_{T, i}\right)$, we may fix $\lambda_{N, i}=1$, so (20) - (25) is equivalent to (13) - (18). Summing (19) for all $i$ shows that (19) is a sufficient condition for (12). By continuity, since (9) holds on $\mathcal{F}$, it also holds on $\mathcal{U}$. By summing (9) with (19), we have also have sufficient conditions for (10) on $\mathcal{U}$.

(19) - (25) may seem stricter than the original formulation when multiple contacts are active. However, by Assumption 1, if (12) holds in any state $(q, v)$, then for each active contact, (19) must hold on some sequence converging to $(q, v)$ and, by continuity, must also hold at $(q, v)$.

Theorem 4 provides a reduced set of conditions, where each condition depends on at most one contact force indeterminate, $\lambda_{T, i}$. These concise conditions will be used throughout the remainder of the paper to generate stability certificates. Furthermore, (20) - (25) restrict $\lambda_{T, i}$ to be within a compact region, whereas, by the original constraints, the forces are unbounded since $\lambda_{N}$ could be scaled arbitrarily. Not only is it practically useful that the feasible set be compact, with respect to numerical tolerances, but compactness also has theoretical consequences for SOS-based methods [43].

\section{Extension to Three Dimensions}

For clarity of presentation, we have focused this discussion on the planar case. While the examples in this work treat two dimensions, the extension to the full three dimensional case, where $\lambda_{T, i} \in \mathbb{R}^{2}$ and $J_{f, i} \in \mathbb{R}^{2 \times n}$, is straightforward and is presented here without proof. Replace conditions (22) - (24) with

$$
\begin{aligned}
\left(J_{f, i} v\right) \circ \lambda_{T, i} & \leq 0, \\
\mu^{2}-\left\|\lambda_{T, i}\right\|^{2} & \geq 0, \\
\left(\mu^{2}-\left\|\lambda_{T, i}\right\|^{2}\right)\left(J_{f, i} v\right) & =0, \\
{\left[\lambda_{T, i}\right]_{1}\left[J_{f, i} v\right]_{2}-\left[\lambda_{T, i}\right]_{2}\left[J_{f, i} v\right]_{1} } & =0,
\end{aligned}
$$

where we take $\circ$ to represent the Hadamard product and $[x]_{i}$ is the $i$ th element of vector $x$. The first and third of these constraints are vector-valued and are treated elementwise. Note that the fourth constraint is new to the three dimensional case, and is used to ensure that the frictional force exactly opposes the direction of motion.

\section{E. Semialgebraic Representation}

To apply tools from algebraic geometry, like SOS programming, it is important that the constraints above be expressible as polynomials. While Taylor approximation of the preceding conditions can always be used, rigid-body dynamics and the manipulator equations offer a great deal of structure that we can exploit to make the problems of control and verification especially amenable to algebraic methods. For many rigid-body systems, especially those of interest in robotics, trigonometric substitutions can reduce the task of kinematics to an algebraic problem [58]. Concretely, for rotational joints, substituting new indeterminates $c_{i}$ and $s_{i}$ for $\cos \left(q_{i}\right)$ and $\sin \left(q_{i}\right)$ respectively, with the constraint that $s_{i}^{2}+c_{i}^{2}=1$, will often result in polynomial kinematics in $c_{i}$ and $s_{i}$. Prismatic (or translational) joints, require no such substitution and naturally result on polynomial kinematics. Most common robotic joints can be represented as a sequence of such rotational and prismatic transformations (see [48], Sec. 1.4), and so this polynomial representation can be easily formed. Helical joints, however, are a notable exception, since a single helical joint creates both rotational and translational motion. For simple contact surfaces, such as between a point contact and the ground, the gap function and Jacobians $\phi, J$, and $J_{f}$ are kinematic functions, and thus polynomial in $c_{i}, s_{i}$ and the remaining translational coordinates of $q$.

Similarly, the various terms of the manipulator equations and constraints $(H, C$, and $B)$ are also polynomial in the same position coordinates and the standard velocity vector $v$ (see [48], Ch. 2). Several methods can be used to accommodate the appearance of $H(q)^{-1}$ in the conditions of the previous section. First we note that by explicitly introducing an additional variable $\dot{v} \in \mathbb{R}^{n}$, the condition (5) is algebraic in $\dot{v}, v, \lambda$, the translational components of $q$ and any introduced trigonometric variables. Alternatively, as $H(q)$ is positive definite and polynomial, its inverse is a rational function, where the denominator is the $\operatorname{det}(H)$, and thus strictly positive. Therefore, we can find equivalent conditions by multiplying by the denominator. These facts imply that semialgebraic conditions can be posed that are equivalent to those in Section III-B.

\section{APPROACH}

For our systems of interest, the Lyapunov conditions in Section III amount to non-negativity constraints on polynomials over basic semialgebraic sets. This formulation is amenable to SOS-based techniques, which provide certificates that a polynomial can be written as a sum of squares of polynomials, a clearly sufficient condition for non-negativity. Searching over polynomials which satisfy these sufficient conditions can be cast as an SDP, allowing for the application of modern convex optimization tools. For the examples in this paper we use the YALMIP [25], [26] and SPOT [32] toolboxes to generate programs for the semidefinite solvers SeDuMi [52] and MOSEK [34]. For a portion of our approach, we exploit bilinear alternation (related to the techniques of DKiteration [24] and also referred to as coordinate-wise descent). We briefly review these concepts in Appendix C.

\section{A. Global Verification}

For some dynamic systems, we can verify the Lyapunov conditions over the entire admissible set. Define $\mathcal{D}_{i}$ to be the set of all $\left(q, v, \lambda_{i}\right)$ that satisfy the conditions (20) - (25). Note that this also implies $(q, v) \in \mathcal{A}$. If $(0,0)$ is an equilibrium of the system, we can then pose the global feasibility SOS 
program:

find $V(q, v)$

subj. to $V(0,0)=0$,

$$
\begin{array}{lr}
V(q, v) \geq \alpha(\|q\|+\|v\|) & \text { for }(q, v) \in \mathcal{A}, \\
\nabla V^{T}\left[\begin{array}{l}
v \\
\dot{v}
\end{array}\right] \leq 0 & \text { for }(q, v) \in \mathcal{A}, \\
\frac{\partial V}{\partial v} H^{-1}\left(J_{i}^{T}+J_{f, i}^{T} \lambda_{T, i}\right) \leq 0 & \text { for }\left(q, v, \lambda_{i}\right) \in \mathcal{D}_{i},
\end{array}
$$

with $\alpha(\cdot)$ is in class $\mathcal{K}$. SOS allows us to search over a family of polynomial Lyapunov functions via SDP. By finding such a function, we verify that every sublevel set of $V$ is positively invariant and that the origin is stable in the sense of Lyapunov. This certificate of a nested set of invariant regions is weaker than asymptotic stability but stronger than invariance of a single set.

\section{B. Regional Verification}

For many problems of interest, we would like to maximize the verified region about an equilibrium. Specifically, we aim to find a Lyapunov function that maximizes the volume of a connected component $\mathcal{C} \subseteq \Omega_{1} \cap \mathcal{A}$, which is positively invariant and, for all $\rho \leq 1, \mathcal{C} \cap \Omega_{\rho}$ is also positively invariant. This leads to the following optimization problem:

$$
\begin{array}{clr}
\max _{V} & \operatorname{Volume}(\mathcal{C}) & \\
\text { subj. to } & & \\
& & \\
& V(q, 0)=0, & \\
& \nabla V^{T}\left[\begin{array}{c}
v \\
\dot{v}
\end{array}\right] \leq 0 & \text { for }(q, v) \in \mathcal{C}, \\
& \frac{\partial V}{\partial v} H^{-1}\left(J_{i}^{T}+J_{f, i}^{T} \lambda_{T, i}\right) \leq 0 & \text { for }(q, v) \in \mathcal{C}, \\
& & \text { for }\left(q, v, \lambda_{i}\right) \in \mathcal{D}_{i} \\
& \text { and }(q, v) \in \mathcal{C} .
\end{array}
$$

As currently posed, this problem is not amenable to convex optimization techniques. It is difficult to directly measure the volume of $\mathcal{C}$ and, as $\mathcal{C}$ is only one connected component of $\Omega_{1} \cap \mathcal{A}$, it is not naturally described as a semialgebraic set. We approximate these regions by finding polynomials $g_{I}(q, v)$ and $g_{O}(q, v)$ such that their one sublevel sets $\left(\mathcal{G}_{I}\right.$ and $\mathcal{G}_{O}$ resp.) are inner and outer approximations of $\mathcal{C}$, i.e.

$$
\left(\mathcal{G}_{I} \cap \mathcal{A}\right) \subseteq \mathcal{C} \subseteq\left(\mathcal{G}_{O} \cap \mathcal{A}\right)
$$

By containing $\mathcal{C}$ within the semialgebraic set $\mathcal{G}_{O}$ and verifying the Lyapunov conditions on $\mathcal{G}_{O}$, we provide sufficient conditions on $\mathcal{C}$. The inner approximation $\mathcal{G}_{I}$ is used to estimate the volume of the verified region. In practice, we parameterize $g_{I}$ and $g_{O}$ as quadratic forms. For $g_{I}(q, v)=$ $\left[\begin{array}{ll}q^{T} & v^{T}\end{array}\right] G_{I}\left[\begin{array}{ll}q^{T} & v^{T}\end{array}\right]^{T}$, we will use $-\operatorname{Trace}\left(G_{I}\right)$ as a proxy for the volume of $\mathcal{C}$. Given this, we pose the problem:

$$
\begin{aligned}
& \min _{V, G_{I}, G_{O}} \operatorname{Trace}\left(G_{I}\right) \\
& \text { subj. to } V(0,0)=0, \quad G_{I}, G_{O} \succeq 0 \text {, } \\
& V(q, v) \geq \alpha(\|q\|+\|v\|) \quad \text { for }(q, v) \in \mathcal{A} \cap \mathcal{G}_{O}, \\
& \nabla V^{T}\left[\begin{array}{l}
v \\
\dot{v}
\end{array}\right] \leq 0 \\
& \frac{\partial V}{\partial v} H^{-1}\left(J_{i}^{T}+J_{f, i}^{T} \lambda_{T, i}\right) \leq 0 \quad \text { for }\left(q, v, \lambda_{i}\right) \in \mathcal{D}_{i} \\
& V(q, v) \geq 1 \\
& \text { and }(q, v) \in \mathcal{G}_{O} \text {, } \\
& \text { for }(q, v) \in \mathcal{A} \\
& \text { and } g_{O}(q, v)=1 \text {, } \\
& g_{I}(q, v) \geq 1 \\
& \text { for }(q, v) \in \mathcal{A} \backslash \Omega_{1} \text {. }
\end{aligned}
$$

This problem verifies the Lyapunov conditions on the outer approximation $\mathcal{G}_{O}$ and ensures the containment in (32). It is now posed in the familiar form of an optimization over polynomials that are positive on a basic semialgebraic set. As described in Appendix $\mathrm{C}$, we use a bilinear alternation technique to solve this problem. One of the potentially difficult aspects of this alternation is that we must typically supply an initial feasible Lyapunov candidate. Previous sums-of-squares based methods have used local linearizations of the dynamics to find initial candidates [55], [53], but this approach fails when the dynamics are discontinuous. Instead, we have used two potential methods for determining an initial Lyapunov function. The simplest strategy is to use the observation that the passive rigid-body dynamics and inelastic collisions are energetically conservative, and that taking $V$ to be the total energy provides a viable starting point for most mechanical systems. Alternatively, bilinear alternations can be initiated by choosing candidates for $G_{I}$ and $G_{O}$, such as ellipsoids with $\mathcal{G}_{I}$ small and $\mathcal{G}_{O}$ relatively large. In the examples in this work, we will generally take the later approach, to avoid initializing the alternations at a particular Lyapunov candidate, and thus biasing towards energy as a solution.

Solutions to (33) are guaranteed to be feasible Lyapunov functions to the original problem (31), although they will generally be suboptimal. This method, however, provides a tractable technique to synthesize useful regional certificates through contact discontinuities. An alternate approach to bilinear alternations is to fix $\mathcal{G}_{O}$ and to fix the form of $\mathcal{G}_{I}$ to within a scalar factor and pose (33) as a feasibility problem. The optimal scaling of $\mathcal{G}_{I}$ can then be found by binary search. Though it only searches over a subset of the solutions to the first formulation, this SDP may be better conditioned numerically for some applications.

\section{Safety}

The algorithm above for verifying stability and invariance can be easily adapted to address questions of dynamic safety. For instance, we might wish to determine the largest set of initial conditions such that the infinite horizon reachable set does not intersect some unsafe semialgebraic set $\mathcal{X}_{u}$. We pose this problem in a manner similar (33), although here we do 
not require that $V$ be positive definite:

$\min _{V, G_{I}, G_{O}} \operatorname{Trace}\left(G_{I}\right)$

subj. to $G_{I}, G_{O} \succeq 0$,

$$
\begin{array}{lr}
\nabla V^{T}\left[\begin{array}{l}
v \\
\dot{v}
\end{array}\right] \leq 0 & \text { for }(q, v) \in \mathcal{A} \cap \mathcal{G}_{O}, \\
\frac{\partial V}{\partial v} H^{-1}\left(J_{i}^{T}+J_{f, i}^{T} \lambda_{T, i}\right) \leq 0 & \text { for }\left(q, v, \lambda_{i}\right) \in \mathcal{D}_{i} \\
V(q, v) \geq 1 & \text { and }(q, v) \in \mathcal{G}_{O}, \\
& \text { for }(q, v) \in \mathcal{A} \\
V(q, v) \leq 1 & \text { and } g_{O}(q, v)=1, \\
V(q, v) \geq 1 & \text { for }(q, v) \in \mathcal{A} \cap \mathcal{G}_{I}, \\
& \text { for }(q, v) \in \mathcal{A} \cap \mathcal{X}_{u} .
\end{array}
$$

The optimization program in (34) verifies that $\mathcal{C}$ is positively invariant and that $\mathcal{C} \cap \mathcal{X}_{u}=\emptyset$, so no trajectory that originates in $\mathcal{C}$ can leave the safe region.

\section{Control Design}

This approach to Lyapunov analysis of autonomous systems can be naturally extended to the design of feedback control laws, as detailed in [18]. The method was later extended to verify stability along trajectories and then experimentally tested in [28]. Traditionally applied to continuous systems, here we consider the problem of designing a polynomial feedback law $u(q, v)$ that is smoothly dependent on state. The use of such a control law, would provide robustness to disturbances that may induce unexpected impacts and to uncertainties in sensing the contact state. Design of a hybrid or switching controller would additionally suffer from the same combinatorial mode enumeration issues faced by verification of such models. When controlling a robotic system near to multiple contact surfaces, a stable, mode-invariant feedback law would be both robust and simple to implement.

For control input $u$ and (possibly state-dependent) matrix $B$, the continuous dynamics are given in the standard controlled manipulator form

$$
H(q) \dot{v}+C(q, v)=B(q, v) u+J(q)^{T} \lambda_{N}+J_{f}(q)^{T} \lambda_{T} .
$$

The task of feedback design can then be expressed as finding control law $u(q, v)$ such that the conditions in Theorem 4 holds. This amounts to a modification of (9) to be

$$
\nabla V(q, v)^{T}\left[\begin{array}{c}
v \\
H^{-1}(B u(q, v)-C)
\end{array}\right] \leq 0 .
$$

Since both $V(q, v)$ and $u(q, v)$ are optimization parameters, this constraint is bilinear in $V$ and $u$, and so, here too we employ an alternating method. When merged with the regional verification of Section IV-B, the problem remains bilinear, and so a two-step alternation approach suffices.

The full process detailing initialization and iterations for control design and regional verification is detailed in Algorithm 1. In ITERATIONA, we fix $G_{I}$ and the Lyapunov function, up to a scale factor, and we search for the scale factor, the control policy $u(q, v)$, and $G_{O}$. Conversely, in ITERATIONB, we fix $G_{O}$ and the control policy, and we search for the Lyapunov function and $G_{I}$. By splitting the decision parameters in this manner, each alternation is convex and can be represented by a SDP. Furthermore, the described modifications for global analysis and safety are straightforward to implement in practice.

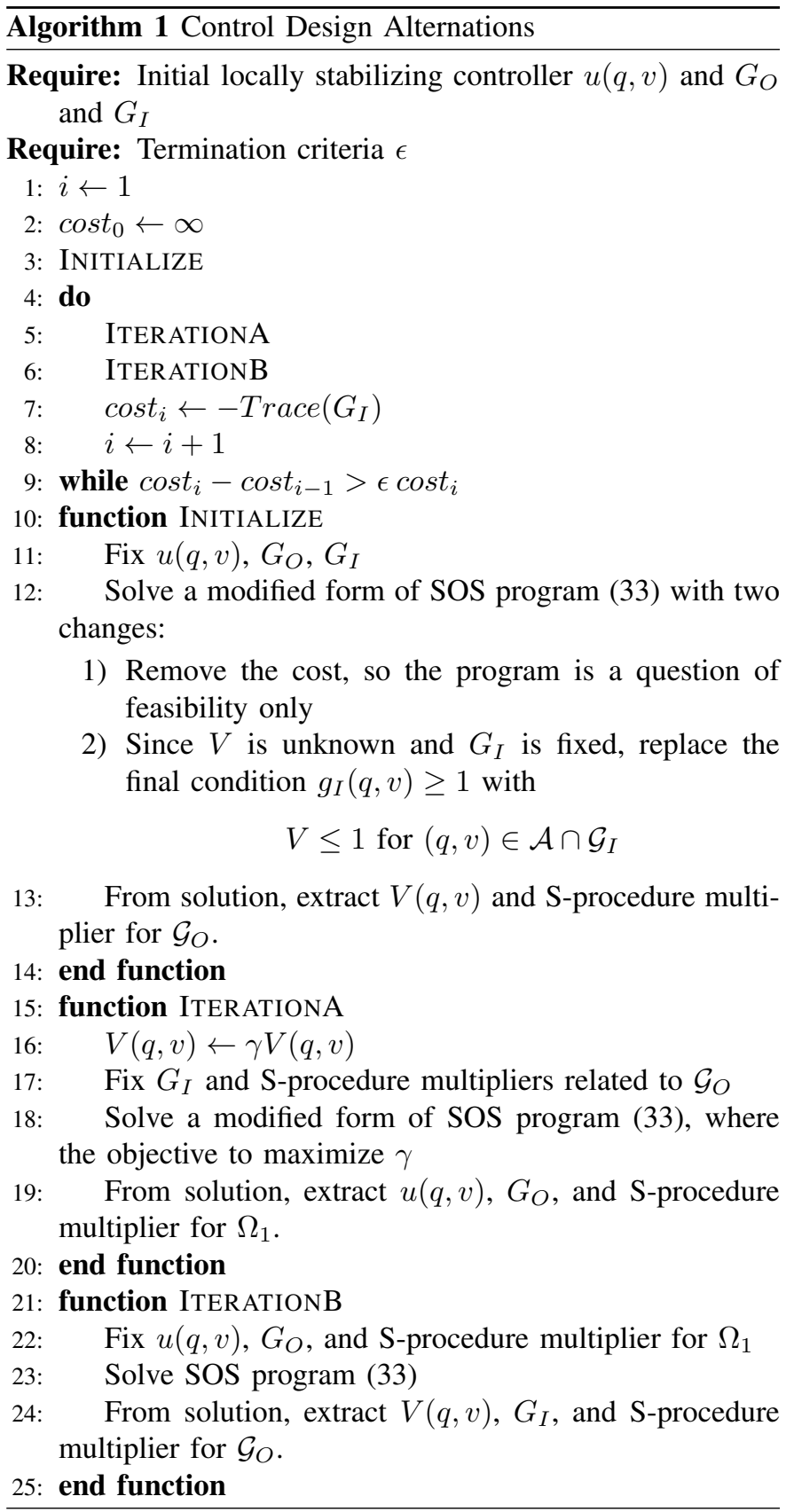

\section{E. Complexity}

To numerical precision, SDPs can be efficiently solved in practice and theoretically solved in polynomial time [57]. The difficulty of these problems is dependent on the number of variables and semidefinite constraints. Let $n$ be the dimension of the system state space, $m$ the number of potential contacts, and $d$ the total degree of the polynomial representation used. Then, the approaches presented in this section construct SOS programs with $\mathcal{O}(m)$ non-negativity constraints to verify that 
$\mathrm{d} V \leq 0$. The inequality (25) results in the generation of $\mathcal{O}(m)$ S-procedure multipliers per such constraint, each of which is transformed into a semidefinite constraint of size $\mathcal{O}\left((n+1)^{d}\right)$ in the SDP. Therefore, there are $\mathcal{O}\left(m^{2}\right)$ semidefinite constraints, and $\mathcal{O}\left(m^{2}(n+1)^{d}\right)$ variables in the SDP. Note that had we not used the formulation from Theorem 4 and decoupled the contact constraints, the SDP size would be $\mathcal{O}\left(m(n+m)^{d}\right)$. By comparison, hybrid formulations that enumerate each mode and transition will introduce variables and constraints that scale exponentially in $m$.

\section{EXAMPLES}

\section{A. Bean Bag Toss}

We first examine the simple problem of a bean bag, modeled as a planar point mass, colliding inelastically with the ground. This example serves to demonstrate the method on a system simple enough where the calculations can be easily verified by hand. With $q=\left[\begin{array}{ll}y & z\end{array}\right]^{T}$ and $v=\left[\begin{array}{ll}\dot{y} & \dot{z}\end{array}\right]^{T}$, we define $\phi(q)=z$. The dynamics are given by

$$
m \ddot{y}=\lambda_{T}, \quad m \ddot{z}=-m g+\lambda_{N} .
$$

For this simple system, the dynamics are invariant under $y$ so we consider the equilibrium set where the mass rests on the ground, $\left\{(y, z) \in \mathbb{R}^{2}: z=0\right\}$. Choosing our Lyapunov candidate function be equal to the total energy of the system, we will show stability in the sense of Lyapunov and invariance of a series of nested sets. That is, each sublevel set of energy is positively invariant. Substituting $V(q, v)=E=\frac{1}{2} m \dot{y}^{2}+$ $\frac{1}{2} m \dot{z}^{2}+m g z$ and the dynamics into (30), we have the two conditions:

$$
\begin{array}{r}
-\nabla V^{T}\left[\begin{array}{c}
v \\
\dot{v}
\end{array}\right]=-m g \dot{z}+m g \dot{z} \geq 0 \text { for }(q, v, \lambda) \in \mathcal{D}, \\
-\frac{\partial V}{\partial v} H(q)^{-1}\left(J(q)^{T} \lambda_{N}+J_{f}(q)^{T} \lambda_{T}\right)= \\
-\dot{y} \lambda_{T}-\dot{z} \lambda_{N} \geq 0 \text { for }(q, v, \lambda) \in \mathcal{D} .
\end{array}
$$

The first condition is trivially true. Observing that $J v=\dot{z}$ and $J_{f} v=\dot{y}$, we use $\mathrm{S}$-procedure type multipliers to verify the second condition. Generating sums-of-squares multipliers $\sigma_{i}(q, v, \lambda)$ for the relevant unilateral constraints (15) and (16), replace (37) with

$$
-\dot{y} \lambda_{T}-\dot{z} \lambda_{N}+\sigma_{1} \dot{y} \lambda_{T}+\sigma_{2} \dot{z} \lambda_{N} \text { is SOS. }
$$

Choosing $\sigma_{1}=\sigma_{2}=1$, the equation above vanishes and is trivially non-negative. Thus, we have used our methods to demonstrate the rather obvious fact that every sublevel set of energy will be positively invariant. Note that the quartic Lyapunov function

$$
V=E+E^{2}+\frac{1}{2} \dot{z}^{3}+\frac{1}{2} z \dot{z}
$$

satisfies (30) but where we can additionally verify that $\dot{V}<$ $-\alpha\left(z+\dot{y}^{2}+\dot{z}^{2}\right)$ for some class $\mathcal{K}$ function $\alpha$. Combined with the condition that $\mathrm{d} V \leq 0$, this is sufficient to verify asymptotic stability of the equilibrium set, though we do not prove this here. In general, it is difficult to find such Lyapunov functions for discontinuous mechanical systems.

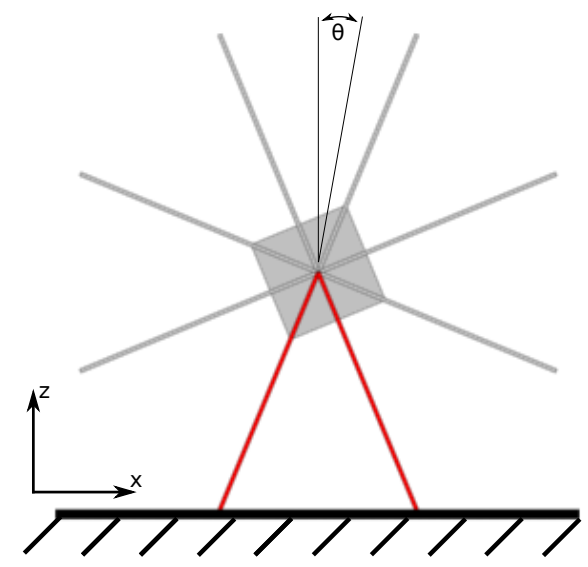

Fig. 2: The rimless wheel shown in an equilibrium state, with two feet on the ground. Verified trajectories pass through four possible contact states (no contact, double-support, and both single-support phases).

\section{B. Rimless Wheel}

The rimless wheel model is a single rigid body composed of a number of equally-spaced spokes about a simple mass. This simple model has been used extensively as a proxy for a passive-dynamic walking robot [10]. Though previous works have primarily been interested in analyzing the limitcycle behavior of the rimless wheel, here we focus on the stability of a single, static configuration of the system. We allow for frictional contacts between two of the spokes and the ground, highlighted in Fig. 2, and we consider the equilibrium set where both of these spokes rest on a flat ground. We differentiate between resting on these two particular spokes and any other equilibrium state. Trajectories of the rimless wheel that come to rest in the equilibrium set may undergo an infinite number of collisions rocking back and forth between the two feet, in an example of Zeno phenomena.

The planar floating base model of the rimless wheel has three degrees of freedom, $q=\left[\begin{array}{lll}x & z & \theta\end{array}\right]^{T}$ and $v=$ $\left[\begin{array}{lll}\dot{x} & \dot{z} & \dot{\theta}\end{array}\right]^{T}$. With the trigonometric substitutions $s=\sin (\theta)$ and $c=\cos (\theta)$, the dynamics of the rimless wheel and the contact related elements $\phi_{i}(q), J_{i}(q)$, and $J_{f, i}(q)$ are all polynomial functions of the redundant state variables $(x, z, s, c, \dot{x}, \dot{z}, \dot{\theta})$ and the contact forces $\left(\lambda_{N, i}, \lambda_{T, i}\right)$. As with the point mass example, the dynamics are invariant under $x$ and so the equilibrium set is defined as $\left\{(x, z, \theta) \in \mathbb{R}^{3}: z=\right.$ $0, \theta=0\}$.

Fixing $g_{i}$ and $g_{o}$, find an initial candidate Lyapunov function as in Algorithm 1. We then use iterations search for a solution to (33) to find a nested set of invariant regions and verify stability in the sense of Lyapunov. When we parameterize $V$ as a quartic polynomial, we verify a significant region of state space about the origin. A slice of this region is shown in Fig. 3a where the verified region is the connected component of $\Omega_{1} \cap \mathcal{A}$ containing the origin. Fig. 3b illustrates the use of $\mathcal{G}_{I}$ and $\mathcal{G}_{O}$ to provide inner and outer bounds on $\mathcal{C}$. 


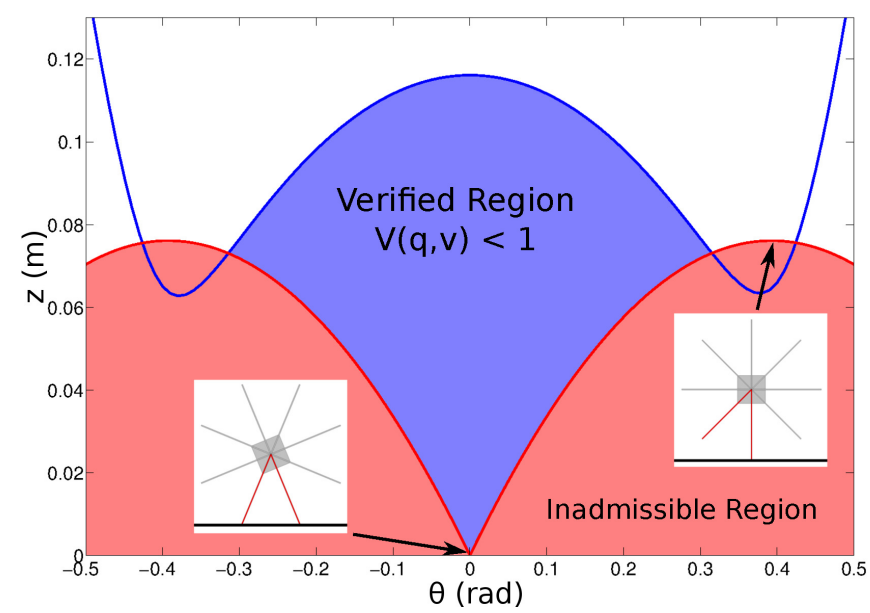

(a) The red region below the solid line indicates the inadmissible set, where at least one of the contact points is penetrating the ground. For reference, two particular states are indicated: the stable equilibrium in double-support at the bottom and an unstable equilibrium in singlesupport on the upper right. The blue region below the dashed line is the connected component $\mathcal{C} \subseteq \Omega_{1} \cap \mathcal{A}$ that contains the equilibrium (the verified region).

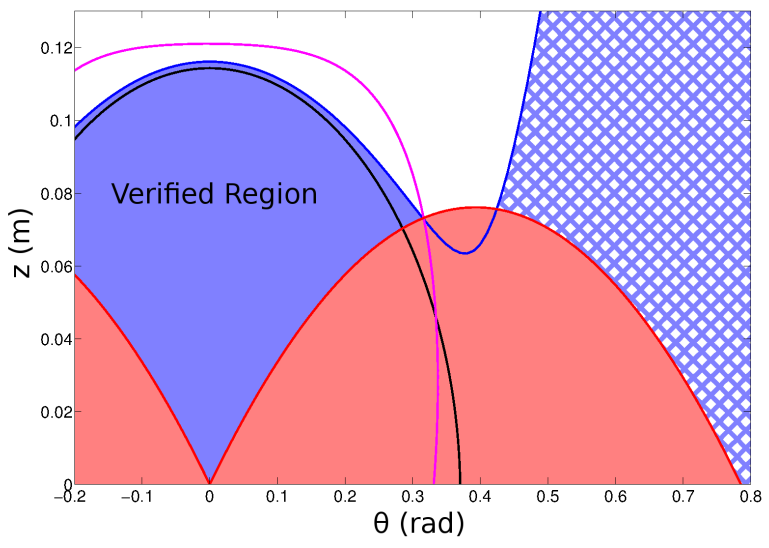

(b) Two additional curves are shown. Under the black curve is the boundary of $\mathcal{G}_{I}$, tight to $\Omega_{1} \cap \mathcal{A}$, which we use to approximate the volume of the verified region. Under the magenta curve is $\mathcal{G}_{O}$, which must contain $\mathcal{C}$. $\mathcal{G}_{O}$ is parameterized as an ellipsoid in the redundant state variables, including $s$ and $(1-c)$, which is why it is not ellipsoidal when plotted against $\theta$. The hatched region, while a subset of $\Omega_{1} \cap \mathcal{A}$, is not connected to $\mathcal{C}$ and is unverified by our algorithm (and also outside the true invariant region).

Fig. 3: A slice of the rimless wheel state space where all velocities are zero.

It is interesting to note that if we parameterize $V$ as a quadratic polynomial, the alternations quickly converge to verify a region that is identical to the maximal sublevel set of energy that does not contain any additional equilibrium points. Additionally, we recover a scaled version of energy as our Lyapunov candidate. The quartic parameterization, however, verifies a larger region with a Lyapunov function significantly different from energy.

Note that the true region of attraction of this model is unbounded. For instance, for any $x, z$, take $q=\left[\begin{array}{lll}x & z & 0\end{array}\right]^{T}$ and all velocities to be zero. A trajectory starting from any

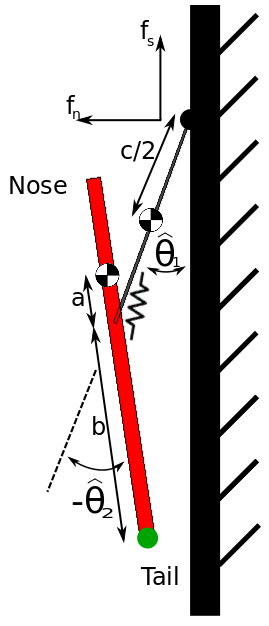

Fig. 4: A simple model of a perching aircraft using two rigid links. The foot of the aircraft is pinned to the wall surface and there is a contact point at the tail that can collide with and slide along the wall. A torsional spring damper connects the main body of the aircraft to the foot.

height will fall and then come to rest in the equilibrium set. By our parameterizations of $\mathcal{G}_{I}$ and $\mathcal{G}_{O}$, our regional approach is limited to ellipsoidal volumes and so will not recover the entire region of attraction. We do find a significant volume about the equilibrium set that would be relevant to planning or control applications.

\section{Perching Glider}

We also examine the problem of verifying a safe set of initial conditions for a glider perching against a wall, by adapting a model first presented in [12]. We consider the instant after the glider feet, which have adhesive microspines, have first impacted the wall and so we treat this contact as a pin joint. The glider is then modeled as a two-link body, with a spring damper connecting the bodies as shown in Fig. 4. We allow the tail of the glider to impact the surface of the wall and slide along it. The specific problem of verification was earlier addressed in [13], although the authors there used a model with a single joint and fixed the tail of the glider to slide along the wall, disallowing collisions. In this paper, we verify a significantly larger region than in our previous work, though a direct comparison is impossible since our model is higher dimension and uses Coulomb friction instead of viscous damping at the tail contact.

There are two relevant failure modes for the perching behavior of the glider, described in more detail in [13]. In one, the nose of the glider impacts the wall, which would be a potentially damaging event. In the other, the force limit of the feet microspines is exceeded and the glider falls from the wall. The force at the feet is a rational function of the state variables, and so the force limit can be easily expressed as a semialgebraic constraint.

This is a two degree of freedom model, with $q=\left[\begin{array}{ll}\hat{\theta}_{1} & \hat{\theta}_{2}\end{array}\right]^{T}$ and we again use a trigonometric substitution for both angles. We also change coordinates so that $(0,0)$ is an equilibrium 


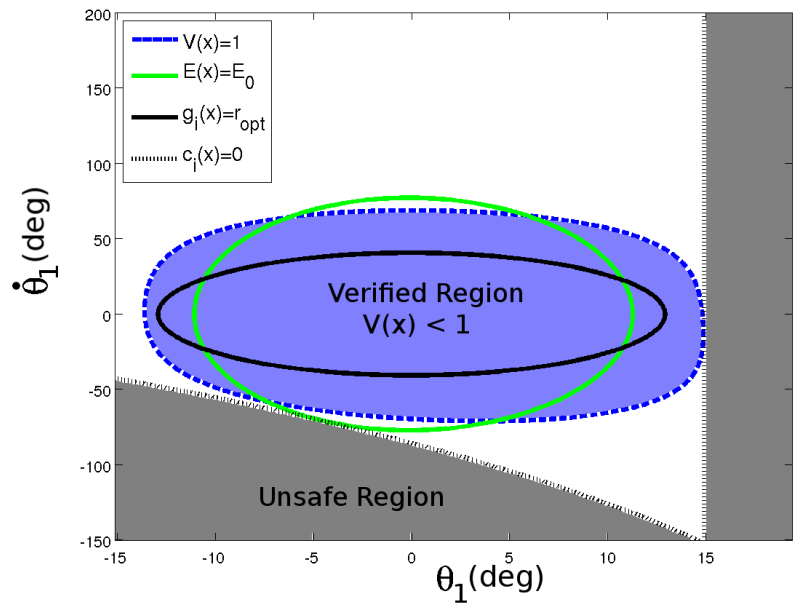

(a) A slice of the glider state space where the tail is restricted to the surface of the wall. The shaded blue region within the dashed line indicates the verified region $\mathcal{C} \cap \mathcal{A}$ for a quartic Lyapunov function and the solid black ellipse outlines $\mathcal{G}_{I}$. The green ellipse indicates the maximal sublevel set of energy that does not intersect the unsafe region, shown in gray.

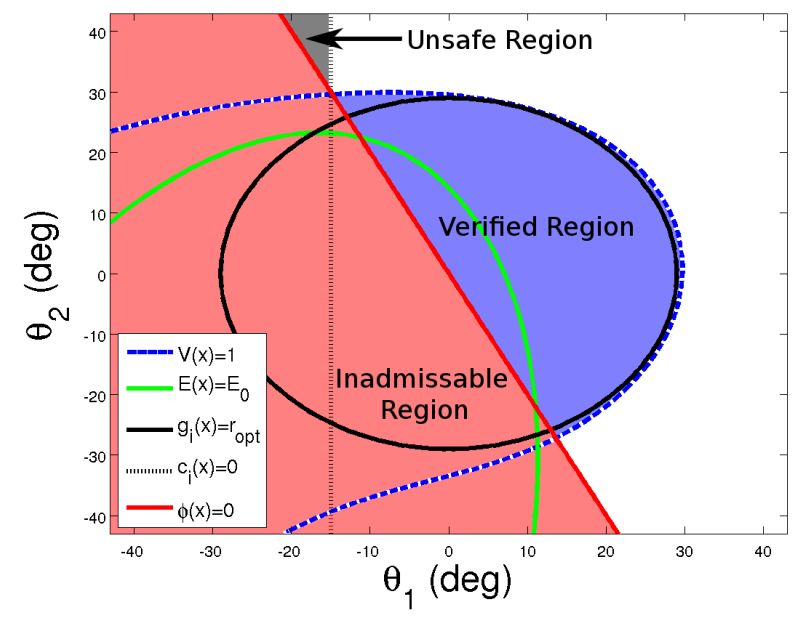

(b) A second slice of state space where the joint velocities are zero is also shown. In this slice, the general quartic Lyapunov function vastly outperforms energy and $\mathcal{G}_{I}$ is tight to $\mathcal{C}$.

Fig. 5: Two slices of the glider state space

point with the tail resting against the wall, substituting $\theta_{1}=$ $\hat{\theta}_{1}-0.2604$ and $\theta_{2}=\hat{\theta}_{2}+0.5207$. With the rimless wheel, $H(q)$ was a constant matrix and so $H^{-1}(q)$ was also constant. Here, $H(q)^{-1}$ is rational and so, for our dynamical constraints, we clear the denominator to ensure that our conditions are algebraic.

We search for a solution to (34), to maximize the set of initial conditions of trajectories that do not violate either constraint. Letting $\mathcal{G}_{O}$ be all of $\mathbb{R}^{2 n}$, define

$$
\mathcal{G}_{I}(\rho)=\left\{(q, v): 2\left(1-c_{1}\right)+2\left(1-c_{2}\right)+0.1\|v\|^{2}<\rho\right\} .
$$

We seek to maximize $\rho$ through a binary search, observing that $2\left(1-c_{i}\right)$ well approximates $\theta_{i}^{2}$ near $\theta_{i}=0$. As with the rimless wheel, if we restrict our search to parameterizations of $V$ of

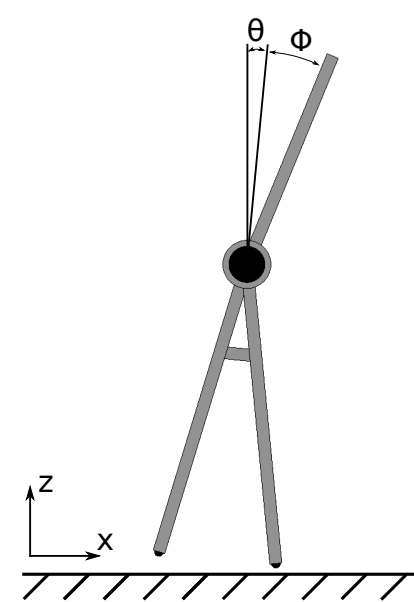

Fig. 6: A simple balancing robot with a rigid base and actuated torso. Two feet can make contact with the ground, in a manner similar to the rimless wheel.

equal degree to energy, we recover the maximal sublevel set of energy that does not intersect either constraint boundary. If we expand our search to include all quartic polynomials, we find a Lyapunov function which verifies a visibly larger region. Two slices of this region are visualized in Fig. 5a and 5b. Our binary search terminates finding $\rho=0.25$ and we can verify that an upper bound on the true optimal value is $\rho=$ 0.327 , since there $\mathcal{G}_{I}(\rho)$ intersects the constraint boundary. Of course, the true optimal value might be lower still since there is no claim that any $\mathcal{G}_{I}(\rho)$ is invariant. Here, we find a significant invariant region that usefully approximates the safe set of initial conditions.

\section{Balancing Robot Control Design}

Lastly, we present a control design example that builds on the rimless wheel. The system here is a simple balancing robot described by planar dynamics. The lower body is similar to that of the rimless wheel, where the two legs are at a fixed angle, each with a single contact point at the end. The upper mass is connected to the lower body by a single actuated joint. When both feet are motionless with respect to the ground, the robot resembles an inverted pendulum. In all other contact configurations, it has varying degrees of underactuation. As with the rimless wheel, the dynamics are invariant in the $x$ direction, and so we can treat the robot as a 7 state system. As with previous examples, the balancing robot also undergoes Zeno phenomena. Typical simulated trajectories undergo a lengthy sequence of contact transitions, including single and double support phases as well as sliding modes.

For this example, we use cubic Taylor expansions of the dynamics and contact constraints. Algorithm 1 is initialized with a simple PD controller on $\phi$ and $G_{i}$ and $G_{o}$ such that both locally approximate total energy. Note that because of the unilateral constraints, and the linear dependence of gravitational potential on height, the Hessian of energy must be modified slightly to form this approximation. For optimizing the verified region, the controller was parameterized as a full state feedback cubic controller. A quartic function was used 
to parameterize the Lyapunov candidates, and S-procedure multiplier degrees were chosen to balance the total degree of each sums-of-squares condition.

Using SPOT to generate the SDP and MOSEK to solve it, each iteration of Algorithm 1 (two SOS programs) took approximately 45 minutes. For $\epsilon=.01$, convergence was reached in 30 iterations. While this total running time is substantial, the complexity is dominated by the dimensionality of the plant, not the number of contact points. For comparison, each iteration for rimless wheel, which has two fewer states and no actuation, runs in approximately 90 seconds on a desktop machine. As with the other examples, the algorithm is able to verify a significant region of state space surrounding the equilibrium, slices of which are illustrated in Figure 7. In a 7 dimensional state space, a true experimental evaluation of the stabilized region is intractable. Similar to the rimless wheel, the true stabilized region is unbounded in some directions. In other slices, however, sampling the space and simulating gives a rough indication that the verified region is a good approximation of the true stabilized region, illustrated in Figure $7 b$.

\section{CONCLUSIONS AND FUtURE WORK}

Reliable planning and control through contact, as in locomotion and manipulation tasks, are critical challenges in robotics. The natural structure of rigid-body dynamics and the complementarity formulation of frictional impacts provide a framework for posing questions of stability and invariance as sums-of-squares optimization problems. This paper presents a class of algorithms for numerical computation of Lyapunov certificates for such systems, as well as for the design of modeinvariant stabilizing controllers. By invoking the complementarity model of contact, we avoid directly reasoning about both the complexity of Zeno phenomena and the combinatorial number of hybrid modes associated with the set of potential contact states. Initial experiments in a simulated environment have found physically significant certificates of stability and invariance for multiple problems of interest to the robotics community.

In this work, we have been primarily interested in stability in the sense of Lyapunov and positive invariance, but we hope to extend these methods to asymptotic stability. One challenge with this extension is that friction often leads to a connected set of equilibria, which poses difficulties for methods based on analytic Lyapunov functions. That aside, two common approaches to verifying asymptotic stability are to find a Lyapunov candidate where $V$ is strictly negative or to apply LaSalle's Invariance Principle. With the former method, energy no longer provides an initial feasible candidate to begin bilinear alternation. In [22], Theorem 6.31 gives a generalization of LaSalle to discontinuous systems with the additional condition that the limit sets of trajectories also be positively invariant. The algorithms presented here might be extended to meet this condition and search for certificates of asymptotic stability.

Future work in this area will also center on extending these algorithms to more complex tasks. While the examples in

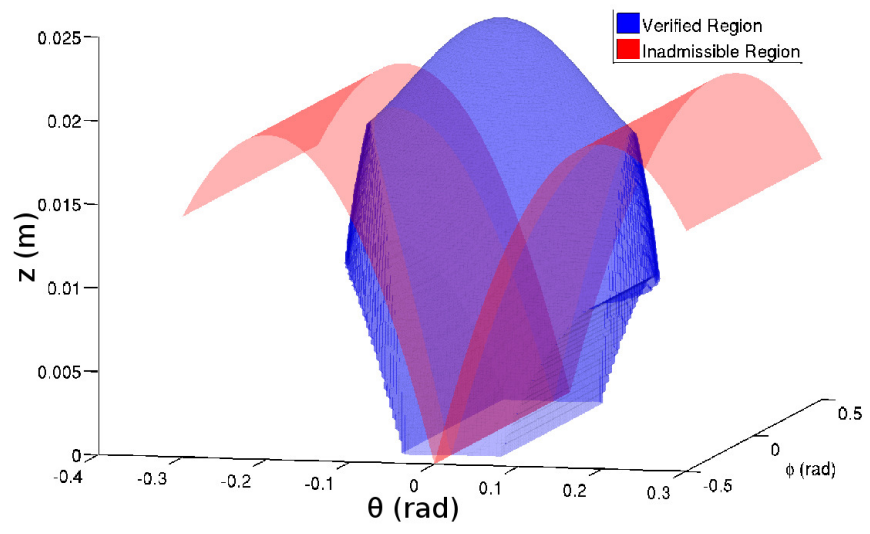

(a) A three dimensional slice of the state space, where all velocities have been set to zero. Here, the blue region is the verified volume, although, in the figure, it extends slightly past the contact surfaces for viewing purposes.

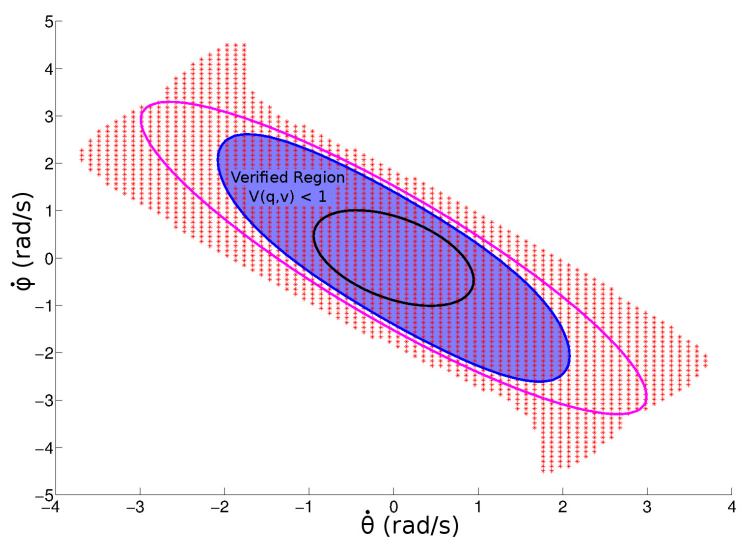

(b) A second slice is shown with respect to the angular velocities, where positions and translational velocities set to zero. This figure additionally illustrates the boundaries of $\mathcal{G}_{i}$ and $\mathcal{G}_{o}$ in in black and magenta. With initial conditions in this slice, trajectories were simulated by a time-stepping method ([50]) to find the experimental region of attraction. The trajectories that converged to the equilibrium are indicated with red stars.

Fig. 7: Two slices of robot the state space.

this paper are relatively low dimensional, commercial SDP solvers are relatively immature and are rapidly improving. Additionally, recent work on hierarchical relaxations of SOS problems have shown promise in solving significantly larger problems [29], [21]. In particular, we are interested in the analysis of trajectories and limit cycles of robotics systems, where previous work has demonstrated the effectiveness of SOS-based methods [31]. By extending to trajectory analysis, we might verify the stability of locomotion gait primitives [14] or that of walking motions with respect to terrain variations [45]. Mode-invariant analysis might also be applied to methods which reduce the dimensionality of walking problems, such as the form of zero dynamics explored in [19]. Furthermore, a natural extension of this work would be to include elastic impacts, where many models exist which are amenable to complementarity formulations such as in [51]. Recent work on the computation of regions of attraction for polynomial 
systems has included convex, moment based approaches [16], with extensions to hybrid systems [46] and control design [30]. A similar approach here might eliminate the requirement for numerically challenging bilinear alternations and allow the problem to be posed as a single convex optimization program.

\section{ACKNOWLEDGMENTS}

This work was supported by the National Science Foundation [Contract IIS-1161679], Defense Advanced Research Projects Agency program [BAA-10-65-M3-FP-024], and the Rolf Locher Graduate Fellowship.

\section{APPENDIX A}

PRoOF OF THEOREM 2

Proof. Fix a solution $x(t)=(q(t), v(t))$ with $x^{-}\left(t_{0}\right) \in \mathcal{C}$. Let $\bar{\tau}$ be the supremum over all $\tau$ such that $x^{-}(t) \in \mathcal{C}$ for all $t \in\left[t_{0}, \tau\right]$. Assume for contradiction that $\bar{\tau}$ is finite. We see that $x^{-}(\bar{\tau}) \in \mathcal{C}$ as it is the limit of a sequence in a connected component. The function $s \mapsto(q(\bar{\tau}), \bar{v}(s))$ provides a path from $x^{-}(\bar{\tau})$ to $x^{+}(\bar{\tau})$. This path lies in $\Omega_{c}$ as $\mathrm{d} V \leq 0$ on $\mathcal{C}$ implies $V\left(x^{-}(\bar{\tau})\right) \leq V\left(x^{-}\left(t_{0}\right)\right)<c$ and, by assumption, $V(q(\bar{\tau}), \bar{v}(s))$ is a non-increasing function of $s$. The path lies in $\mathcal{A}$ as only the generalized velocities vary (recall our definition of $\mathcal{A}$ in (3)). Thus $x^{+}(\bar{\tau})$ belongs to $\mathcal{C}$. As $V\left(x^{+}(\bar{\tau})\right)<c$ and $V$ is continuous, there exists an $r>0$ such that:

$$
U_{r}=\left\{x \in \mathbb{R}^{2 n}:\left\|x-x^{+}(\bar{\tau})\right\|_{\infty}<r\right\}
$$

is contained in $\Omega_{c}$ (where $\|\cdot\|_{\infty}$ is the maximum norm). As $x^{+}(\tau)$ is a right limit, there exists an $\epsilon>0$ such that $x(t) \in U_{r}$ for $t \in(\bar{\tau}, \bar{\tau}+\epsilon)$.

We show that $x(t) \in \mathcal{C}$ for almost all $t \in(\bar{\tau}, \bar{\tau}+\epsilon)$, contradicting the definition of $\bar{\tau}$. Fix $t$ such that $v(t)$ is defined and examine the following functions:

$$
\begin{aligned}
& \sigma_{1} \mapsto\left(q\left(\sigma_{1}\right), v^{+}(\bar{\tau})\right), \\
& \sigma_{2} \mapsto\left(q(t), \sigma_{2} v(t)+\left(1-\sigma_{2}\right) v^{+}(\bar{\tau})\right),
\end{aligned}
$$

the first defined for $\sigma_{1} \in[\bar{\tau}, t]$ and the second for $\sigma_{2} \in[0,1]$. Since $q(\cdot)$ is continuous, both functions are also continuous. We see the range of both maps lie in $\mathcal{A}$ as $\{q(t)\} \times \mathbb{R}^{n} \subset \mathcal{A}$ for all $t \geq t_{0}$. We see the ranges of the functions also lie in $U_{r}$ : the first lies in $U_{r}$ as $\left(q_{1}, v_{1}\right),\left(q_{2}, v_{2}\right) \in U_{r}$ implies $\left(q_{2}, v_{1}\right) \in U_{r}$ and the second as $U_{r}$ is convex. Together, these functions provide a path from $x^{+}(\bar{\tau})$ to $x(t)$ that lies in $\Omega_{c} \cap \mathcal{A}$, thus $x(t) \in \mathcal{C}$.

\section{APPENDIX B}

\section{PROOF OF PROPOSITION 3}

Proof. That (ii) implies (i) is the content of Theorem 2. Now assume (i) holds and fix a solution $(q(t), v(t))$ with $\left(q(t), v^{-}(t)\right) \in \mathcal{C}$. For convenience, let $q, v^{+}$, and $v^{-}$denote $q(t), v^{+}(t), v^{-}(t)$. Take the path $\bar{v}(s)=s v^{+}+(1-s) v^{-}$. Since $V$ is convex in $v$ and $\mathrm{d} V \leq 0$, we know

$$
V\left(q, v^{-}\right) \geq(1-s) V\left(q, v^{-}\right)+s V\left(q, v^{+}\right) \geq V(q, \bar{v}(s)),
$$

so that the chord $(q, \bar{v}(s))$ lies in $\Omega_{c}$, and clearly lies in $\mathcal{A}$.
We show that $\frac{d V(q, \bar{v}(s))}{d s} \leq 0$. Observe that $\{(q, \bar{v}(s): s \in$ $[0,1)\}$ are all possible pre-impact states since $\phi(q)=0$ and the impact conditions $J v^{-}<0, J v^{+}=0$ imply that $J \bar{v}(s)<0$. Let $\Lambda_{N}$ be a feasible impulse such that $v^{+}=v^{-}+H^{-1} J^{T} \Lambda_{N}$. Substituting into the definition of $\bar{v}(s)$,

$$
\bar{v}(s)=v^{-}+s H^{-1} J^{T} \Lambda_{N} .
$$

Since the constraints on $\Lambda_{N}$ are linear, we know that the impulse $(1-s) \Lambda_{N}>0$ will also be feasible. Applying this impulse to $(q, \bar{v}(s))$ we get the post-impact velocity

$$
\bar{v}^{+}(s)=\bar{v}(s)+(1-s) H^{-1} J^{T} \Lambda_{N}=v^{+} .
$$

And so $v^{+}$is a possible post-impact velocity from an impact at any point along the chord. Since $\mathrm{d} V \leq 0$, we then know $V(q, \bar{v}(s)) \geq V\left(q, v^{+}\right)$. This implies that the minimum of $V$ along the chord is achieved at $\left(q, v^{+}\right)$and, since $V$ is convex, the derivative along the chord must be non-positive.

\section{APPENDIX C \\ S-PROCEDURE AND BILINEAR ALTERNATION}

Sum-of-squares methods enable optimization over linearly parameterized polynomials that are guaranteed to be nonnegative. This can be extended to guarantee non-negativity on basic semialgebraic sets in a process generally referred to as the S-procedure [37]. To demonstrate that $g(x) \geq 0$ implies $f(x) \geq 0$ we introduce multiplier polynomials $\sigma_{i}(x)$ and require:

$$
\sigma_{1}(x) f(x)-\sigma_{2}(x) g(x) \geq 0, \quad \sigma_{1}(x)-1 \geq 0, \quad \sigma_{2}(x) \geq 0 .
$$

In general, we may wish to simultaneously search over the free coefficients of the multiplier polynomials $\sigma_{i}(x)$ and those of $f(x)$ and $g(x)$. However, such coefficients enter the condition in a bilinear fashion. Fixing the free coefficients of $f$ and $g$, we can search over the free coefficients of $\sigma_{1}$ and $\sigma_{2}$, and vice versa. These allows for a coordinate-descent strategy where each step consists of a single convex optimization problem and is guaranteed to improve the objective value. Similar approaches have been applied to determine region-of-attraction estimates for smooth dynamical systems [55].

In practice, bilinear alternations have the possibility of failing due to numerical conditioning. Many of the SDPs generated from SOS programs have no feasible interior, due to an overparameterization of the the sums-of-squares basis. This issue is referred to as facial reduction, and recent work has been done to enhance the typical pre-processing steps to reduce the dimensionality of the SDP [38]. However, no tractable general solution exists for solving the facial reduction problem. With no feasible interior, interior point algorithms can solve each iteration to within some numerical tolerance, but this tolerance, combined with alternations, can lead to infeasible optimization problems. To address this issue, we make two modifications to the standard SPOT and MOSEK algorithms. First, we use a projection based method as a post-processing step to reduce numerical residuals, similar to the accelerated first-order approach of [3]. Second, we use a regularization technique to perturb the SOS program by some small amount, to ensure that a feasible interior exists 
and Slater's condition will hold. This regularization is similar to the recent approach used in [17]. Post-processing and regularization have both been useful in stabilizing the bilinear alternations used throughout this work.

\section{REFERENCES}

[1] R. Alur, T. Henzinger, G. Lafferriere, and G. Pappas. Discrete abstractions of hybrid systems. Proceedings of the IEEE, 88(7):971 -984, July 2000.

[2] A. D. Ames, H. Zheng, R. D. Gregg, and S. Sastry. Is there life after zeno? taking executions past the breaking (zeno) point. In American Control Conference, 2006, pages 6-pp. IEEE, 2006.

[3] D. Bertsimas, R. M. Freund, and X. A. Sun. An accelerated firstorder method for solving SOS relaxations of unconstrained polynomial optimization problems. Optimization Methods and Software, 28(3):424441, 2013.

[4] V. Bhatt and J. Koechling. Three-dimensional frictional rigid-body impact. Journal of Applied Mechanics, 62:893, 1995.

[5] M. Branicky, V. Borkar, and S. Mitter. A unified framework for hybrid control: model and optimal control theory. IEEE Transactions on Automatic Control, 43(1):31 -45, Jan 1998.

[6] B. Brogliato. Nonsmooth mechanics: models, dynamics, and control. Springer Verlag, 1999.

[7] B. Brogliato. Some perspectives on the analysis and control of complementarity systems. IEEE Transactions on Automatic Control, 48(6):918-935, 2003.

[8] C. Cai, A. R. Teel, and R. Goebel. Smooth Lyapunov functions for hybrid systems-part i: existence is equivalent to robustness. IEEE Transactions on Automatic Control, 52(7):1264-1277, 2007.

[9] A. Chatterjee and A. Ruina. A new algebraic rigid-body collision law based on impulse space considerations. Journal of Applied Mechanics, 65(4):939-951, 1998.

[10] M. J. Coleman, A. Chatterjee, and A. Ruina. Motions of a rimless spoked wheel: a simple 3d system with impacts. Dynamics and Stability of Systems, 12(3):139-160, 1997.

[11] S. H. Collins, A. Ruina, R. Tedrake, and M. Wisse. Efficient bipedal robots based on passive-dynamic walkers. Science, 307:1082-1085, February 182005

[12] A. L. Desbiens, A. Asbeck, and M. Cutkosky. Hybrid aerial and scansorial robotics. Proceedings of the 2010 IEEE International Conference on Robotics and Automation (ICRA), pages 72-77, May 2010.

[13] E. L. Glassman, A. L. Desbiens, M. Tobenkin, M. Cutkosky, and R. Tedrake. Region of attraction estimation for a perching aircraft: A Lyapunov method exploiting barrier certificates. In Proceedings of the 2012 IEEE International Conference on Robotics and Automation (ICRA), 2012.

[14] R. D. Gregg, A. K. Tilton, S. Candido, T. Bretl, and M. W. Spong. Control and planning of 3-d dynamic walking with asymptotically stable gait primitives. IEEE Transactions on Robotics, 28(6):1415-1423, 2012.

[15] D. Henrion and A. Garulli, editors. Positive Polynomials in Control. Lecture Notes in Control and Information Sciences. Springer-Verlag, 2005.

[16] D. Henrion and M. Korda. Convex computation of the region of attraction of polynomial control systems. IEEE Transactions on Automatic Control, 59(2):297-312, 2014.

[17] D. Henrion and J. Malick. Projection methods for conic feasibility problems: applications to polynomial sum-of-squares decompositions. Optimization Methods \& Software, 26(1):23-46, 2011.

[18] Z. Jarvis-Wloszek, R. Feeley, W. Tan, K. Sun, and A. Packard. Some controls applications of sum of squares programming. In 42nd IEEE Conference on Decision and Control, volume 5, pages 4676 - 4681, December 2003

[19] J. Lack, M. J. Powel, and A. D. Ames. Planar multi-contact bipedal walking using hybrid zero dynamics. In 2014 IEEE International Conference on Robotics and Automation (ICRA), pages 2582-2588. IEEE, 2014.

[20] A. Lamperski and A. D. Ames. Lyapunov theory for zeno stability. IEEE Transactions on Automatic Control, 2012

[21] J.-B. Lasserre, K.-C. Toh, and S. Yang. A bounded degree SOS hierarchy for polynomial optimization. arXiv preprint arXiv:1501.06126, 2015.

[22] R. Leine and N. van de Wouw. Stability and convergence of mechanical systems with unilateral constraints. Springer Verlag, 2008.

[23] R. Leine and N. van de Wouw. Stability properties of equilibrium sets of non-linear mechanical systems with dry friction and impact. Nonlinear Dynamics, 51(4):551-583, 2008.
[24] R. Lind, G. Balas, and A. Packard. Evaluating D-K iteration for control design. In American Control Conference, 1994, volume 3, pages 2792 - 2797 vol.3, 29 June-1 July 1994.

[25] J. Lofberg. YALMIP: A toolbox for modeling and optimization in MATLAB. In Computer Aided Control Systems Design, 2004 IEEE International Symposium on, pages 284-289. IEEE, 2004.

[26] J. Lofberg. Pre- and post-processing sum-of-squares programs in practice. IEEE Transactions On Automatic Control, 54(5):1007-, May 2009.

[27] J. Lygeros, K. Johansson, S. Simic, J. Zhang, and S. Sastry. Dynamical properties of hybrid automata. IEEE Transactions on Automatic Control, 48(1):2 - 17, Jan 2003.

[28] A. Majumdar, A. A. Ahmadi, and R. Tedrake. Control design along trajectories with sums of squares programming. In Proceedings of the 2013 IEEE International Conference on Robotics and Automation (ICRA), 2013.

[29] A. Majumdar, A. A. Ahmadi, and R. Tedrake. Control and verification of high-dimensional systems with DSOS and SDSOS programming. In Proceedings of the 53rd Conference on Decision and Control (CDC), 2014.

[30] A. Majumdar, R. Vasudevan, M. M. Tobenkin, and R. Tedrake. Convex optimization of nonlinear feedback controllers via occupation measures. International Journal of Robotics Research (IJRR), 33(9):1209-1230, August 2014.

[31] I. R. Manchester. Transverse dynamics and regions of stability for nonlinear hybrid limit cycles. Proceedings of the 18th IFAC World Congress, extended version available online: arXiv:1010.2241 [math.OC], AugSep 2011.

[32] A. Megretski. Systems polynomial optimization tools (SPOT), available online: http://web.mit.edu/ameg/www. 2010

[33] J. J. Moreau. Unilateral contact and dry friction in finite freedom dynamics. In Nonsmooth mechanics and Applications, pages 1-82. Springer, 1988.

[34] A. Mosek. The mosek optimization software. Online at http://www.mosek.com, 54, 2010.

[35] Y. Or and A. Ames. Stability and completion of Zeno equilibria in Lagrangian hybrid systems. IEEE Transactions on Automatic Control, 56(6):1322 -1336, June 2011.

[36] A. Papachristodoulou and S. Prajna. Robust stability analysis of nonlinear hybrid systems. IEEE Transactions on Automatic Control, 54(5):1035 -1041, May 2009.

[37] P. A. Parrilo. Semidefinite programming relaxations for semialgebraic problems. Mathematical Programming, 96(2):293-320, 2003.

[38] F. Permenter and P. Parrilo. Basis selection for SOS programs via facial reduction and polyhedral approximations. Proceedings of the IEEE Conference on Decision and Control, December 2014.

[39] M. Posa, C. Cantu, and R. Tedrake. A direct method for trajectory optimization of rigid bodies through contact. International Journal of Robotics Research, 33(1):69-81, January 2014.

[40] M. Posa, M. Tobenkin, and R. Tedrake. Lyapunov analysis of rigid body systems with impacts and friction via sums-of-squares. In Proceedings of the 16th International Conference on Hybrid Systems: Computation and Control (HSCC 2013), pages 63-72. ACM, April 8-11 2013.

[41] S. Prajna, A. Jadbabaie, and G. Pappas. A framework for worstcase and stochastic safety verification using barrier certificates. IEEE Transactions on Automatic Control, 52(8):1415 -1428, Aug 2007.

[42] S. Prajna and A. Rantzer. Convex programs for temporal verification of nonlinear dynamical systems. SIAM Journal of Control and Optimization, 46(3):999-1021, 2007.

[43] M. Putinar. Positive polynomials on compact semi-algebraic sets. Indiana University Mathematics Journal, 42(3):969-984, 1993.

[44] E. Routh. Dynamics of a system of rigid bodies. MacMillan and co. London, 1891.

[45] C. O. Saglam and K. Byl. Switching policies for metastable walking. In 2013 IEEE 52nd Annual Conference on Decision and Control (CDC), pages 977-983. IEEE, 2013.

[46] V. Shia, R. Vasudevan, R. Bajcsy, and R. Tedrake. Convex computation of the reachable set for controlled polynomial hybrid systems. In Proceedings of the 53rd Conference on Decision and Control (CDC), page 8, 2014.

[47] A. S. Shiriaev and L. B. Freidovich. Transverse linearization for impulsive mechanical systems with one passive link. IEEE Transactions On Automatic Control, 54(12):2882-2888, December 2009.

[48] B. Siciliano and O. Khatib, editors. Handbook of Robotics. Springer, 2008.

[49] D. Stewart. Rigid-body dynamics with friction and impact. SIAM Review, 42(1):3-39, 2000. 
[50] D. Stewart and J. Trinkle. An implicit time-stepping scheme for rigid body dynamics with inelastic collisions and coulomb friction. International Journal for Numerical Methods in Engineering, 39(15):26732691, 1996.

[51] W. Stronge. Rigid body collisions with friction. Proceedings of the Royal Society of London. Series A: Mathematical and Physical Sciences, 431(1881):169-181, 1990.

[52] J. F. Sturm. Using SeDuMi 1.02, a Matlab toolbox for optimization over symmetric cones. Optimization Methods and Software, 11(1-4):625 $653,1999$.

[53] R. Tedrake, I. R. Manchester, M. M. Tobenkin, and J. W. Roberts. LQR-Trees: Feedback motion planning via sums of squares verification. International Journal of Robotics Research, 29:1038-1052, July 2010.

[54] C. Tomlin, I. Mitchell, A. Bayen, and M. Oishi. Computational techniques for the verification of hybrid systems. Proceedings of the IEEE, 91(7):986 - 1001, july 2003.

[55] U. Topcu, A. Packard, and P. Seiler. Local stability analysis using simulations and sum-of-squares programming. Automatica, 44(10):2669 $-2675,2008$.

[56] A. J. van der Schaft and J. Schumacher. Complementarity modeling of hybrid systems. IEEE Transactions on Automatic Control, 43(4):483490, 1998.

[57] L. Vandenberghe and S. Boyd. Semidefinite programming. SIAM Review, 38(1):49-95, 1996.

[58] C. W. Wampler and A. J. Sommese. Numerical algebraic geometry and algebraic kinematics. Acta Numerica, 20:469-567, 2011.

[59] Y. Wang and M. T. Mason. Two-dimensional rigid-body collisions with friction. ASME Journal of Applied Mechanics, 59:635-642, 1992.

[60] E. R. Westervelt, J. W. Grizzle, C. Chevallereau, J. H. Choi, and B. Morris. Feedback Control of Dynamic Bipedal Robot Locomotion. CRC Press, Boca Raton, FL, 2007.

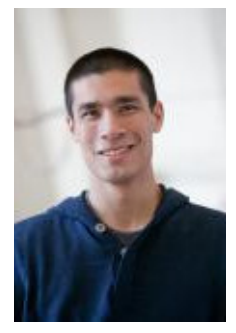

Michael Posa Michael completed his B.S. and M.S. in Mechanical Engineering from Stanford University in 2007 and 2008 and is currently pursuing his Ph.D. in Electrical Engineering and Computer Science at MIT, working with Russ Tedrake in the Robot Locomotion Group. His research emphasizes computational approaches for control and planning of robotic systems with frictional contact. Between his masters and doctoral programs, he worked as a robotics engineer with Vecna Technologies in Cambridge, MA.

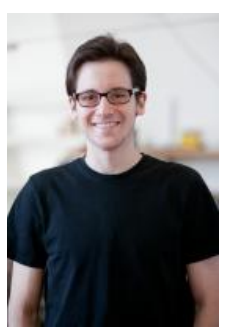

Mark Tobenkin Mark received his S.B. in Computer Science and M.Eng. in Electrical Engineering and Computer Science from MIT in 2009. $\mathrm{He}$ completed his Ph.D. in Electrical Engineering and Computer Science under 2013 studying under Russ Tedrake and Alexandre Megretski. He currently works at Makani Power, a part of Google[x] in Alameda, CA.

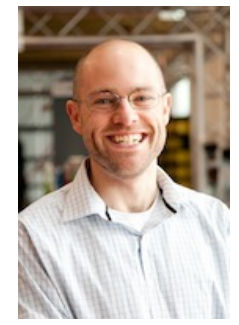

Russ Tedrake Russ is the X Consortium Associate Professor of Electrical Engineering and Computer Science and Aeronautics and Astronautics at MIT, and the Director of the Center for Robotics at the Computer Science and Artificial Intelligence Lab. He is a recipient of the NSF CAREER Award, the MIT Jerome Saltzer Award for undergraduate teaching, the DARPA Young Faculty Award in Mathematics, the 2012 Ruth and Joel Spira Teaching Award, and was named a Microsoft Research New Faculty Fellow. Russ received his B.S.E. in Computer Engineering from the University of Michigan, Ann Arbor, in 1999, and his $\mathrm{Ph} . \mathrm{D}$. in Electrical Engineering and Computer Science from MIT in 2004, working with Sebastian Seung. After graduation, he joined the MIT Brain and Cognitive Sciences Department as a Postdoctoral Associate. During his education, he has also spent time at Microsoft, Microsoft Research, and the Santa Fe Institute. 